\title{
Automated Instruments for In-Line Accounting of Highly Enriched Uranium at the Oak Ridge Y-12 Plant
}

\author{
P. A. Russo \\ R. B. Strittmatter \\ E. L. Sandford \\ M. M. Stephens \\ T. L. Brumfield* \\ S. E. Smith* \\ E. E. McCullough* \\ I. W. Jeter ${ }^{*}$ \\ G. L. Bowers*
}

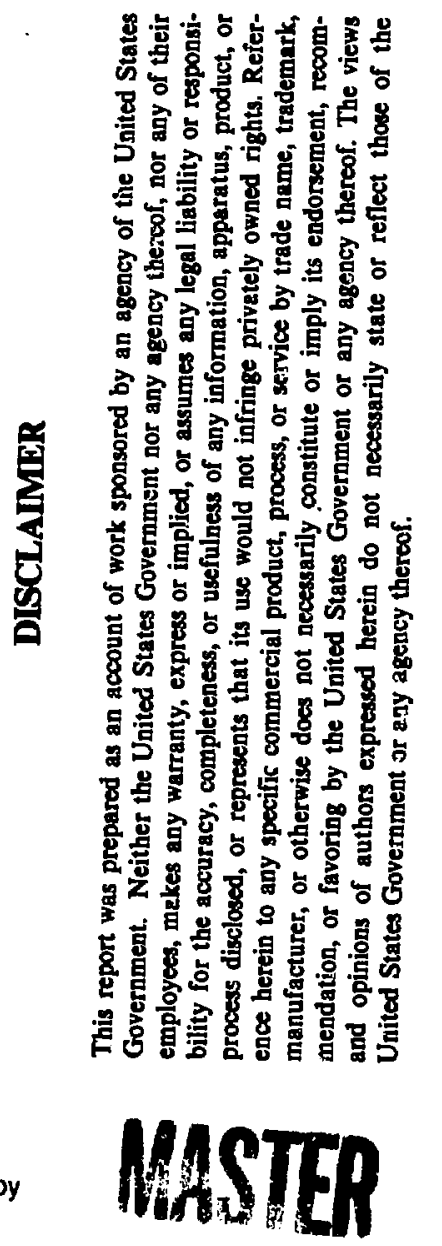

-Oak Ridge Y-12 Plant, Oak Ridge, TN 37830, operated for the US Department of Energy by Martin Marietta Energy Systems, Inc., under Contract DE-AC05-840R20400.

Los Alamos National Laboratory Los Alamos,New Mexico 87545 
CONTENTS

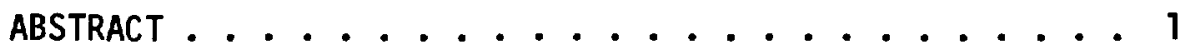

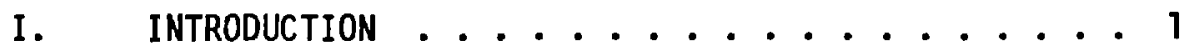

II. INSTRUMENT FUNCTION AND DESIGN ......... 3

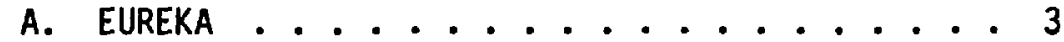

B. Evaporator Monitor ........... 7

C. Uranium Concentration Assay ........ 10

D. Measurement Control ........ 18

E. Results and Significance of Measurement

Control by Uranium Disk Assay . . . . . . 20

III. RESULTS OF SOLVENT EXTRACTION SYSTEM INVENTORY

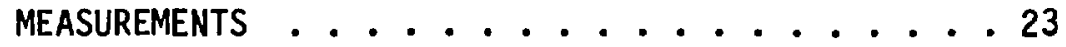

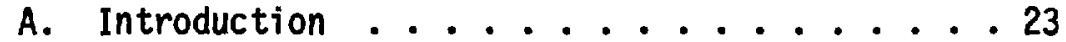

B. Direct Inventory Results ......... 23

C. EUREKA Inventory Results ........ 25

IV. RESULTS OF EVAPORATOR MONITOR EVALUATION .... 33

Y. EVALUATION OF EUREKA RESULTS ........44

A. Column and Pulser Inventories ........ 41

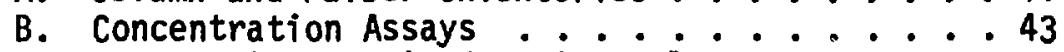

C. Inventories Obtained During Pulsed Operation . 44

D. System Inventory Results .......446

VI. DISCUSSION .................... 47

A. EUREKA ................... 47

1. Improvements in System Inventory ....47

2. Evaluation of the Uncertainty Introduced

by the Histogram Method ....... 53

3. Surmary and Recormendations ...... 54

B. Evaporator Monitor .......... 55

ACKNOWLEDGMENTS .............. 56

REFERENCES ............................. 57 


\section{LIST OF FIGURES}

1. Conceptual illustration of one of two secondary solvent extraction systems for HEU purification at $\mathrm{Y}-12 \ldots \ldots \ldots$

2. The six shielded detectors, mounted on one of the solvent extraction columns in the operating area (left), and the electronics in the remote, controlled-environment area (right) are shown conceptually to illustrate the EUREKA design. The electronics package includes (bottom to top) six stabilized amplifiers, a dual floppy diskatte drive, a computer-based multichannel analyzer with CAMAC minicrate (containing multiplexer-router, $A D C$, high-vol tage supply interface, and clock), and a programmable high-voltage power supply. The portable terminal for control of the assay in the operations area is also shown. ................ 5

3(a). Electronics for EUREkA, assembled before shipment to $Y-12$. This equipment consists of (left, bottom to top) the programmable high-voltage power supply, the stabilized anplifiers, the dual floppy diskette unit, the hard-copy unit, and (right) the programmable multicharinel analyzer with CAMAC minicrate.

3(b). EUREKA electronics installed in the controiled-environment area at $Y-12$. A shielded detector is held in the foreground of the photograph. The terminal for control of the assay in the operations area is shown in front of the hard-copy unit. . . . 6

4(a). Shielded detectors for EUREKA, assembled before shipment to $Y-12$. The uranium (working standard) disks, mounted on the tungsten holders, are shown inserted in the six left-most collimator shields. The portable terminal for control of the assay in the operations area is in the background. . . . . . . 8

4(b). Operator at the $Y-12$ HEU scrap recovery facility is shown positioning a EUREKA detector on the extraction column of the secondary solvent extraction system. ............8

5. Conceptual illustration of uranium concentration monitor installed on the $\mathrm{Y}-12$ secondary intermediate evaporator. The shielded detector is shown mounted on the evaporator solution return loop. The electronics package (right), located $5 \mathrm{~m}$ from the evaporator, consists of (1eft to right at the bottom) a stabilized amplifier, high-voltage supply, ADC, and programmable data acquisition and control (PDAC) unit. The LED display unit is also shown. The capability for automatic control of valves by the PDAC unit, although not currently implemented, is illustrated here. 


\section{LIST OF FIGURES (cont)}

6. The equipment for the uranium concentration monitor for the secondary intermediate evaporator is shown installed at $Y-12$. (a) The electronics components in the environmental enclosure are (left to right) the PDAC unit, the high-voltage power supply, the stabilized amplifier, and the $A D C$. Above the environmental cabinet is the LED display unit. (b) The shielded $\mathrm{NaI}(\mathrm{Tl})$ detector is mounted on the return loop of the secondary intermediate evaporator. ........... 10

7. Drawing (to scale) of a radial cross section of a stainless steel section of a secondary solvent exiraction column in the active region. The detector position with respect to the stainless steel stator rods corresponds to the actual positions of the EUREKA detectors on the solvent extraction columns. The curves labeled $\left(\mathrm{H}_{2} \mathrm{O}\right)_{\mathrm{O}}, \quad\left(\mathrm{HNO}_{3}\right)_{0}$, and $(\mathrm{TBP})_{0}$ mark one mean free path for $186-\mathrm{keV}$ gamma rays in pure water, $\mathrm{ni-}$ tric acid $(4 \mathrm{M})$, and $30 \%$ tributyl phosphate in kerosene. . . . . 11

8. Drawing (to scale) of a longitudinal cross section of a stainless steel section of a secondary solvent extraction column in the active region. The detector position with respect to the sieve plates corresponds to the maximum countrate configuration for $186-\mathrm{keV}$ gamma rays. Refer to Fig. 7 caption for definitions. ................ 11

9. Stainless steel sample cell designed to reproduce the construction and internal configuration of the stainless steel sections of the $Y-12$ secondary solvent extraction columns. The shielded detector is shown mounted on the lower half of tine cell, which is fitted internally with stator rods and

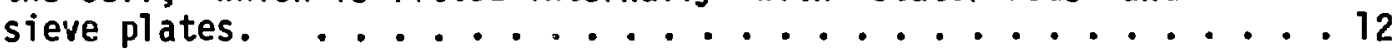

10. The corrected net 186-keV photopeak areas (the 186-keV area corrected for count-rate losses by 60-keV area) measured with the reference detector mounted in different locations on the standard stainless steel sampie cell. Measurements were performed with five different reference solutions of HEU (top). The solid curve is the calculation that applies to the measurements in the inactive (no plates) region (large dots). The ratios of these points to the calculated curve are shown at the bottom. A measurement control data point obtained during each set of solution assays with the reference uranium disk inserted in the collimator is also plotted (asterisks). Measurements in the active region are indicated by crosses (plates only) and triangles (plates and rods). .........13 


\section{LIST OF FIGURES (cont)}

11. The effect of the sieve plates on the $186-\mathrm{keV}$ count rate is illustrated by the data points, which are the ratios of the crosses to the large-dot data points of Fig. 10, plotted vs reference solution concentration. The actual data points shown are each an average of two results: one with the detector collimator positioned as in Fig. 8 and one with the detector collimator positioned between two sieve plates. The error bar limits (upper and lower) show these two results, respectively.

12. The large effect of a stator rod, positioned directly in front of the detector, on the $186-\mathrm{keV}$ colint rate is illustrated by the data points, which are the ratios of the triangular to the cross data points of Fig. 10, plotted vs reference solution concentration. EUREKA avoids these effects by positioning detectors with respect to rods as shown in

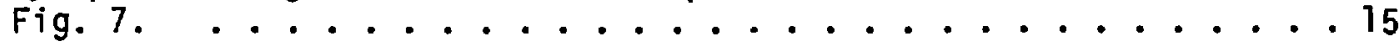

13. The efficiency factor deduced from disk assays (solid points) vs date is plotted for the six EUREKA detectors. With the exception of the points plotted at mid-May and at mid-September, each point since January 1983 corresponds to the average of six disk measurements obtained before solution assays on each of the three columns in the pulsed and static modes during inventory. The dashed lines correspond to the efficiency factors determined by direct measurements, performed in November 1982 at Los Alamos, of reference solutions in the standard cell. The open circles plotted at mid-May and at mid-September are direct measurements performed at $Y-12$ using the standard cell and a uranium reference solution. Disk measurements, which were aiso performed at this time, are plotted as well. The vertical arrows mark the date on which the detector positions were fixed in the shields. . . . . . 21

14. The detector 5 background ( $a$ and $d$ ), foil ( $b$ and $e$ ), and aqueous solution ( $c$ and $f$ ) spectra obtained during the static inventory measurements performed in November 1984. The 60-keV photopeaks are in channels 100 and 290 , respectively. Frames $a, b$, and $c$ show the full spectra, and $d, e$, and $f$ show the upper 300 channels. The data points in the peak regions of interest are intensified. The three background regions of interest (below and above the 60-keV peak and above the 186keV peak) 1 ie between the vertical markers. . . . . . . . 27

15. Correction factor to the detector 6 efficiency factor, deduced empirically for each column, is plotted vs uranium concentration. The mean result and standard deviation are shown for each column by the solid and dashed lines, respectively. Results marked with $X$ were not included in the averaging. 


\section{LIST OF FIGURES (cont)}

16. Uranium concentration plotted vs extraction column height for pulsed and static EUREKA assays at inventory. Smooth curves are drawn to approximate the pulsed and static profiles. The histogram used by the EUREKA code for inventory determination is also shown. Asterisks are external assay results for samples withdrawn from the static aqueous phase at a fixed position. The plotted results correspond to the 1983 inventory measurements after operation for the indicated months. . . . . . 29

17. Uranium concentration plotted vs strip column height for pulsed and static EUREKA assays at inventory. Smooth curves are drawn to approximate the pulsed and static profiles. The histogram used by the EUREKA code for inventory determination is also shown. Asterisks are external assay results for samples withdrawn from the static aqueous phase at a fixed position. The plotted results correspond to the 1983 inventory measurements after operation for the indicated months. . . . . 30

18. Uranium concentration plotted vs backwash column height for pulsed and static EUREKA assays at inventory. Sniooth curves are drawn to approximate the pulsed and static profiles. The histogram used by the EUREKA code for inventory determination is also shown. Asterisks are external assay results for samples withdrawn from the static aqueous phase at a fixed position. The plotted results correspond to the 1983 inventory measurements after operation for the indicated months. . . . . . 31

19. Evaporator monitor results for uranium concentration plotted vs time. The results of the external analysis of two samples wi thdrawn from the evaporator return loop are also plotted at the time of sample withdrawal (solid points). The error bars are calculated from the counting statistics $(1 \sigma)$ of the mon-

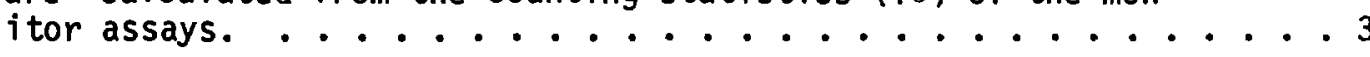

20. Evaporator monitor results for uranium concentration plotted vs time. The results of the external analysis of three samples withdrawn from the evaporator return loop are also plotted at the time of sample withdrawal (solid points). The error bars are calculated from the counting statistics $(1 \sigma)$ of

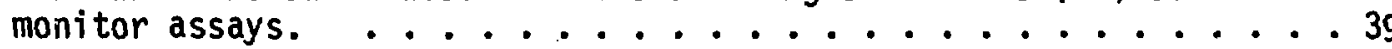

21. Evaporator monitor results for uranium concentration plotted vs time. The results of the external analysis of two samples withdrawn from the evaporator return loop are also plotted at the time of sample withdrawal (solid points). The error bars are calculated from the counting statistics $(1 \sigma)$ of the moni-

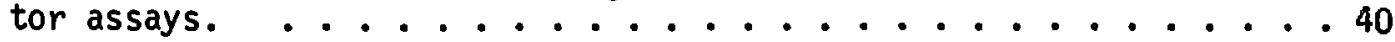




\section{LIST OF FIGURES (cont)}

22. Percentage deviation between evaporator monitor result (measurement) and external sample assay result (tag) plotted vs external sample assay result for the seven withdrawn samples indicated in Figs. 19-21. The error bars are calculated from

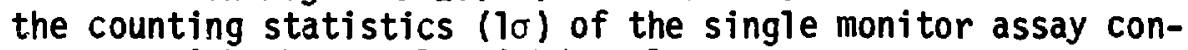
current with the sample withdrawal. 


\section{LIST OF TABLES}

I. Relative random uncertainty $(? \sigma)$ in the 186-keV count rate due to counting statistics and sieve plates ...... 16

II. Relative random uncertainty $(1 \sigma)$ in the $186-\mathrm{keV}$ count rate due to uncertain knowledge of acid molarity .... 17

III. Net relative random uncertainty $(1 \sigma)$ in $186-\mathrm{keV}$ count

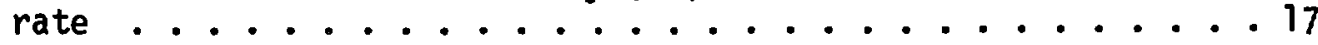

IV. Differential relative uncertainty in uranium concentration versus that in count rate ..........17

V. Relative random uncertainty $(1 \sigma)$ in measured uranium

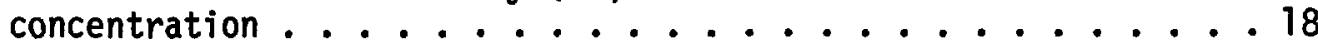

VI. EUREKA detector efficiency factor data deduced from

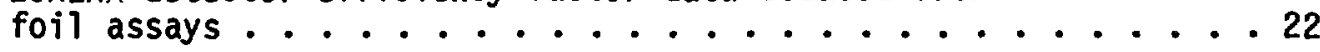

VII. Uranium inventory held up in solvent extraction columns following column dumps

VIII. Dump volumes (milliliters) ............ 25

IX. Dump inventory results $($ grams $U) \ldots \ldots 26$

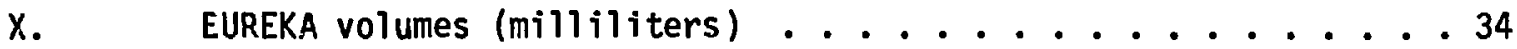

XI. EUREKA inventory results (grams U) ............. 34

XII(A). (EUREKA volume)/(dump volume) ............35

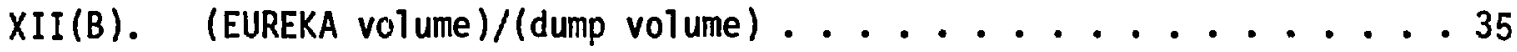

XIII(A). Average values $(\%[\sigma)$ of (EUREKA volume)/(dump volume) . . . . 36

XIII(B). Average values $(\% 1 \sigma)$ of (EUREKA volume)/(dump volume) . . . . 36

XIV(A). (EUREKA inventory)/(dump inventory) .......... 37

XIV(B). (EUREKA inventory)/(dump inventory) .......... 37

XV(A). Average values (\%) $\sigma)$ of (EUREKA inventory)/(dump inventory $) \ldots \ldots . \ldots . \ldots . . \ldots 38$

XV(B). Average values $(\% 1 \sigma)$ of (EUREKA inventory)/(dump inventory $) \ldots \ldots . \ldots . \ldots . \ldots 38$

XVI. EUREKA pulsed and static column inventories (grams $U$ ) .....45

XVII. New plumbing correlations (grams U) ..........48 


\section{LIST OF TABLES (cont)}

XVIII. EUREKA NPC inventory results (grams U) ...........50 $X I X(A)$ (EUREKA NPC inventory)/(dump inventory) .........51 XIX(B). (EUREKA NPC inventory)/(dump inventory) ..........51 $X X(A)$. Average values (\%) $\sigma)$ of (EUREKA NPC inventory)/

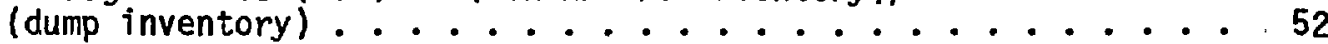

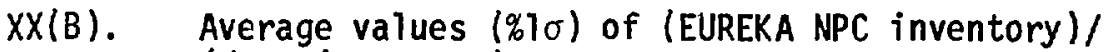

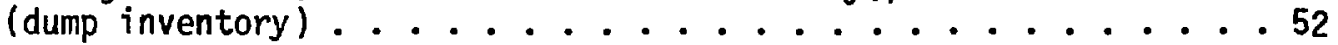

XXI. Inventory (histogram)/inventory (spline) .........54 
AUTOMATED INSTRUMENTS FOR IN-LINE ACCOUNTING OF HIGHLY ENRICHED URANIUM AT THE OAK RIDGE $Y-12$ PLANT

by

P. A. Russo, R. B. Strittmatter, [. L. Sandford, M. M. Stephens, T. L. Brumfield, S. E. Smith, E. E. Mccullough, I. W. Jeter, and G. L. Bowers

\begin{abstract}
Two automated nondestructive assay instruments developed at Los Alamos in support of nuclear materials accounting needs are currently operating in-1ine at the Oak Ridge $Y-12$ facility for recovery of highly enriched uranium (HEU). One instrument provides the HEU inventory in the secondary solvent extraction system, and the other monitors HEU concentration in the secondary intermediate evaporator. Both instruments were installed in December 1982. Operational evaluation: of these instruments was a joint effort of $Y-12$ and Los Alamos personnel. This evaluation included comparison of the solvent extraction system inventories with direct measurements performed on the dumped solution components of the solvent extraction system and comparison of concentration assay results with the external assays of samples withdrawn from the process. The function and design of the instruments and detailed results of the operational evaluation are reported.
\end{abstract}

\title{
I. INTRODUCTION
}

The operation of two automated prototype nondestructive assay (NDA) instruments was evaluated at the 0ak Ridge $Y-12$ facility for highly enriched uranium (HEU) scrap recovery. The instrunents were designed to measure uranium concentrations in-line and to automatically deduce HEU inventories or provide operational information for process control purposes. Each instrument uses a computer-automated data acquisition system to obtain and anaiyze NaI(TI) gammaray spectra characteristic of ${ }^{235} U$, the primary source of radiation in these 
solutions. The instruments were designed ${ }^{1}$ by the Los Alamos National Laboratory Safeguards Assay Group bașed on in-plant NDA measurements using portable equipment ${ }^{2}$ and on recommendations from $Y-12$ Nuclear Materials Control and Accounting (NMC and $A$ ) and Chemical Processing personnel. Operational evaluation was a joint effort of $\mathrm{Y}-12$ and Los Alamos.

These prototype instruments were designed to satisfy two high-priority nuclear materials accounting needs identified by NMC and A. They were

- a timely and reliable method, immune to large biases, to determine the HEU inventory in the secondary solvent extraction system and

- the ability to measure and/or minimize the HEU holdup in the secondary intermediate evaporator that precedes the secondary solvent extraction system in the recovery process.

The methods developed to satisfy these needs, as well as the deveiopment and testing of the methods, had to be compatible with production routines and schedules.

The need for a solvent extraction system inventory is addressed with the Enriched URanium Extraction Kolumn Assay (EUREKA) instrument. EUREKA combines measured concentrations of uranium in the solvent extraction columns (during pulsed operation just before shutdown for inventory and in the static mode after shutdown) with a library of process design information to determine (by use of deduced concentration profiles in the columns) the HEU inventories in the exterrial plumbing and in the columns. The intermediate evaporator holdup problem is addressed with the evaporator monitor, a nonintrusive near-real-time monitor of uranium concentration. This instrument enables more timely manual control of evaporator feed and product flow rates by providing near-real-time concentration information. Although function and design differ substantially in the two instruments, the methods of radiation detection, uranium concentration assay, and measurement control are common to both. Both instruments are nonintrusive on the process.

Operational evaluation of the two prototype instruments began in December 1982 and ended in December 1983. This report reviews the design and functional aspects of these instruments and evaluates the measurement control techniques. The results of the evaluation and the uncertainties in the measurements are presented in detail. A preliminary version of this report ${ }^{3}$ was prepared midway through the operational evaluation. Another report ${ }^{4}$ reviewed the EUREKA evaluation with emphasis on the effectiveness of the instrument for HEU inventory in a production environment. 


\section{A. EUREKA}

The Y-12 facility for recovery of HEU operates two identical, parallel solvent extraction systems for secondary purification of concentrated uranium $(200-\mathrm{g} / l)$ in acid solution $\left(4 \mathrm{MHNO}_{3}\right)$. Figure 1 is a schematic illustration of one of these systems consisting of three pulsed solvent extractior columns, each $10 \mathrm{~m}$ tall by $10 \mathrm{~cm}$ in diameter, with glass (upper one-third) and stainiess steel sections. 5 The glass sections are inactive, and the (active) stainiess steel sections are fitted at $2.5-\mathrm{cm}$ intervals with $1.6-\mathrm{mm}$-thick perforated

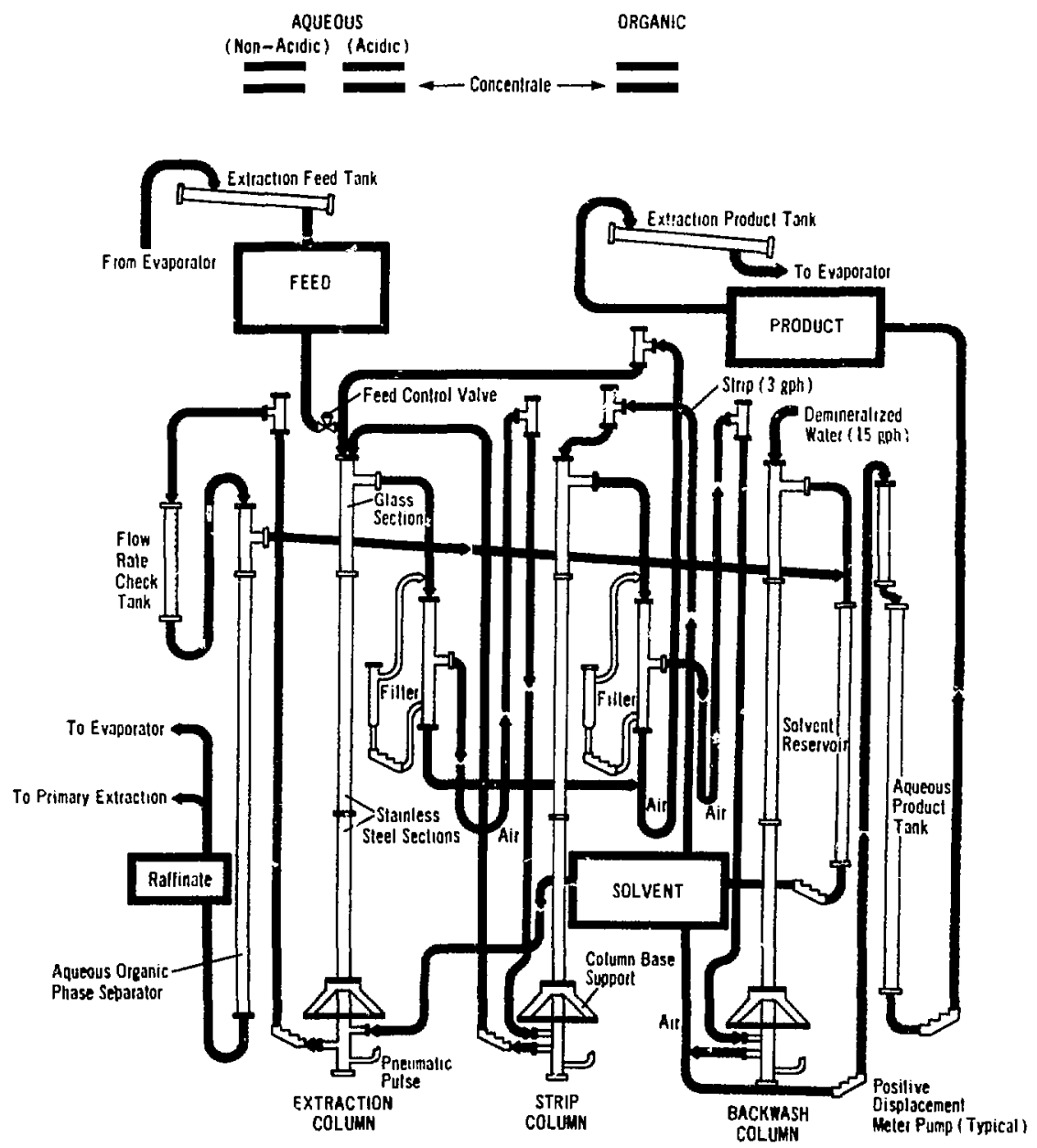

Fig. 1. Conceptual illustration of one of two secondary solvent extraction systems for HEU purification at $Y-12$. 
stainless steel sieve plates for phase dispersion. Uranium is extracted from the acid solutions into the low-density organic phase in the extraction column, scrubbed (of impurities) in the strip column, and stripped from the organic phase into the low-acid aqueous phase in the backwash column. The organic products of the extraction and strip columns are typically saturated $(<120 \mathrm{~g} / \mathrm{l})$ with uranium. The raffinate-stream uranium concentration is a few grams per liter, and the aqueous product concentration is $2100 \mathrm{~g} / \mathrm{l}$. Except for the aqueous feed and product streams and raffinate streams, each system operates in a closed loop. During the monthly shutdown, the aqueous feed and product sturage tanks and the raffinate storage tanks are sampled and assayed for inventory. A separate inventory determination, excluding the storage tanks, is required for the HEU ( $25 \mathrm{~kg}$ per system) in each of the tho parallel solvent extraction systems. This inventory is currently performed on one of the two systems by the EUREKA instrument.

Figure 2 is a conceptual illustration of EUREKA. Six shielded NaI(TI) detectors, mounted for simultaneous acquisition of data along the solutionbearing length $(9 \mathrm{~m})$ of any one of the three columns of the secondary solvent extraction system, are used to determine the uranium concentration at the six vertical locations on each column. The six detectors hang or rest on brackets clamped to (or adjacent to) each column in a fixed position. The detectors are moved from column to column to perform the assays in count times of $600 \mathrm{~s}$. Column inventories are determined from the measured concentrations by construction of a concentration profile for each column in the vertical dimension. The column inventory is the column concentration numerically integrated over the volume el ements.

A11 electronics components downstream from the detectors reside in a remote, controlled-environment location at $Y-12$, as $i 11$ ustrated in Fig. 2. Figure $3(a)$ is a photograph of these electronics assembled before shipment to $Y-12$, and Fig. $3(\mathrm{~b})$ shows the electronics instalied at $Y-12$. The six linear amplifier outputs are multiplexed into a single analog-to-digital converter (ADC) and routed for separate storage in the computer-based multichannel analyzer memory. Analog gain stabilization is employed, and the computer maintains control of the high voltage supplied to each detector. The cathode ray tube display automatically updates any of the six pulse-height spectra in real time (during data acquisition) and provides the option to examine any spectrum in detail after acquisition. The data are automatically analyzed, and the 


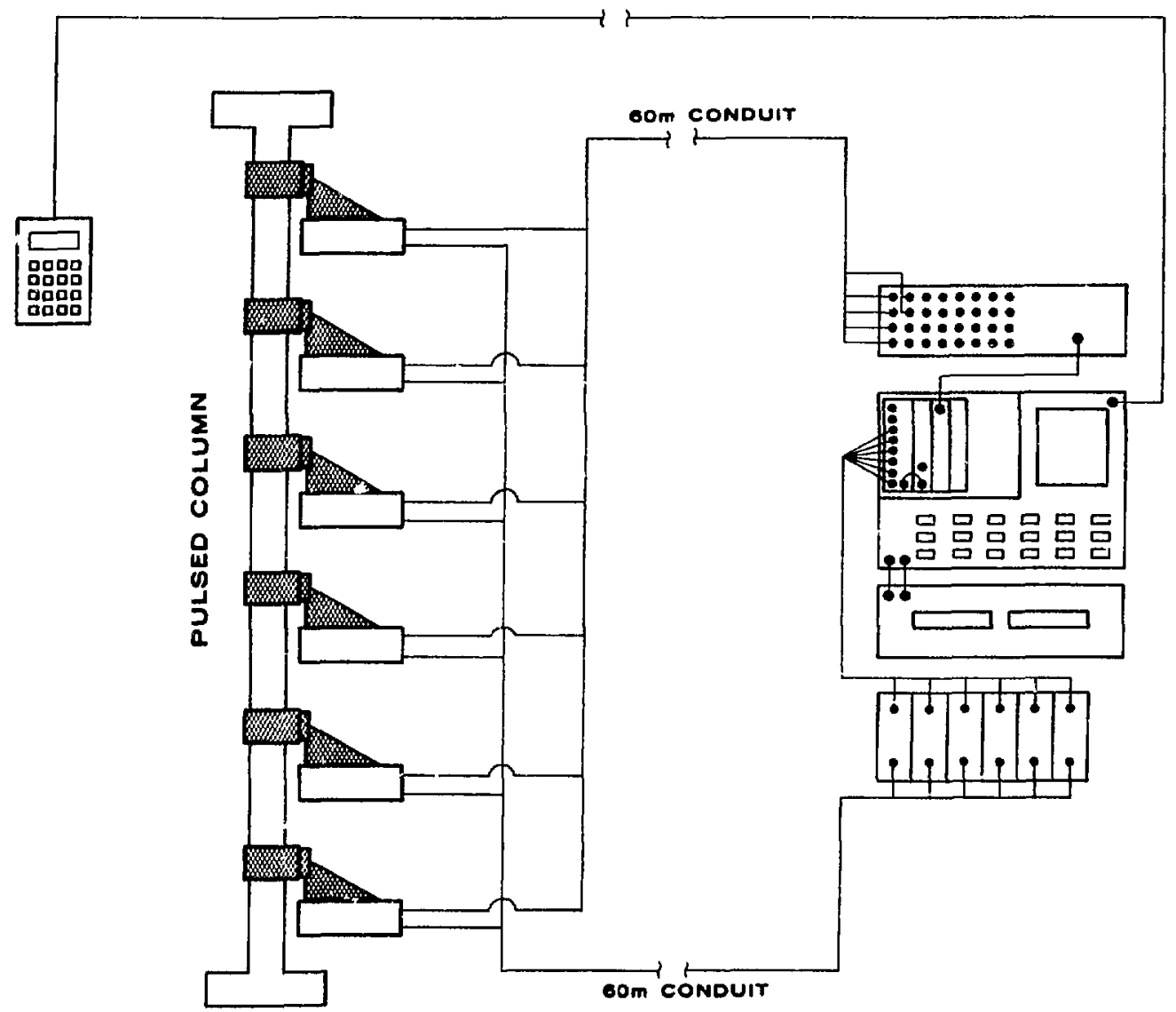

Fig. 2. The six shielded detectors, mounted on one of the solvent extraction columns in the operating area (left), and the electronics in the remote, controlled-environment area (right) are shown conceptually to illustrate the EUREKA design. The electronics package includes (bottom to top) six stabilized amplifiers, a dual floppy diskette drive, a computer-based multichannel analyzer with CAMAC minicrate (containing multiplexer-router, ADC, high-voltage suppiy interface, and clock), and a programmable high-voltage power supply. The portable terminal for control of the assay in the operations area is also shown. 


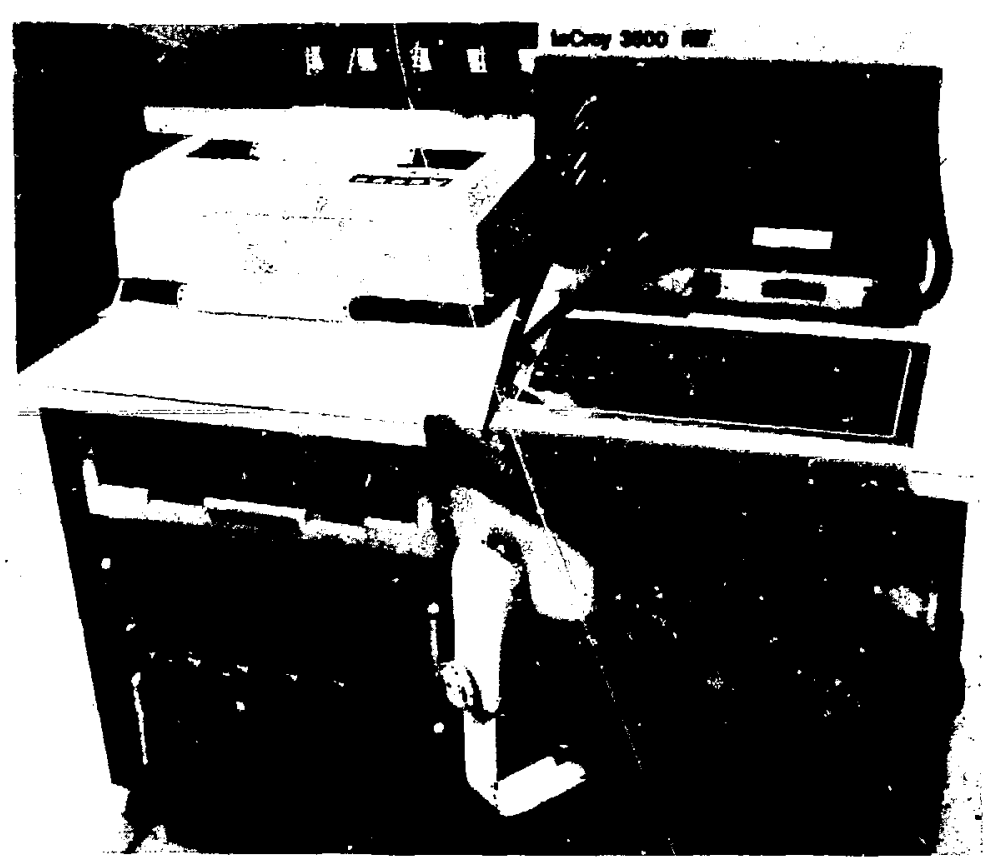

Fig. 3(a). Electronics for EUIREKA, assembled before shipment to $Y-12$. This equipment consists of (1eft, bottom to top) the programmable high-voltage power supply, the stabilized amplifiers, the dual floppy diskette unit, the hard-copy unit, and (right) the programmable multictiannel analyzer with CAMAC minicrate.

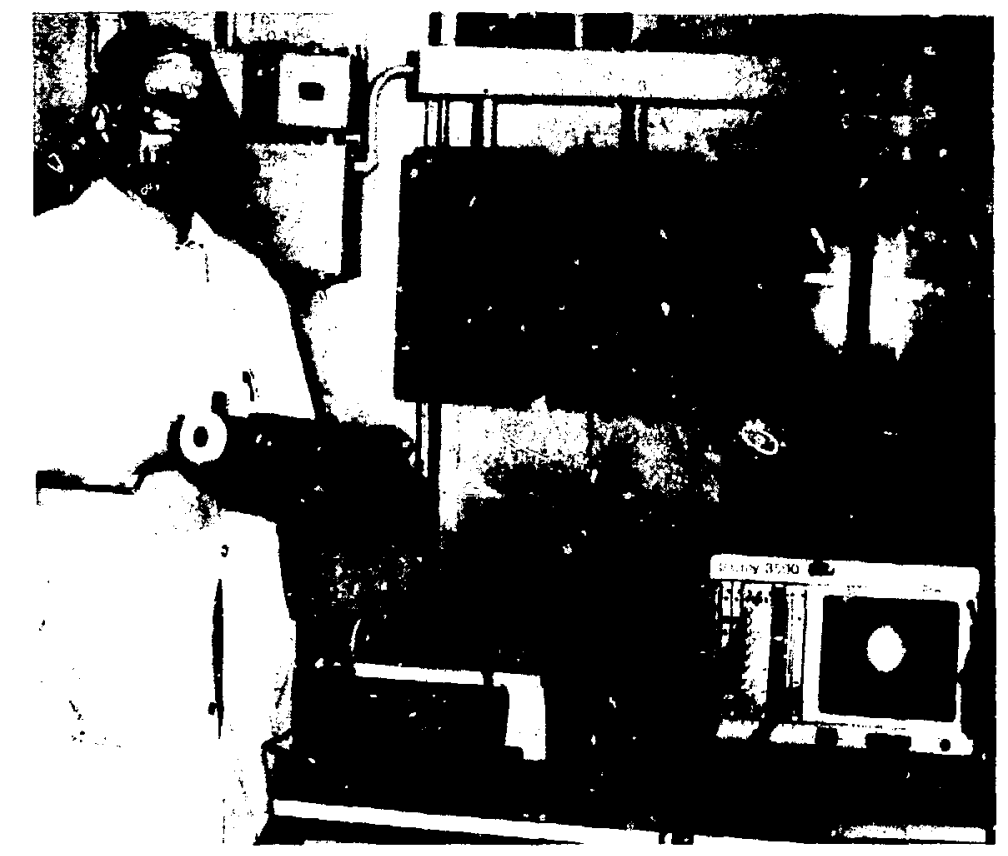

Fig. 3(b). EUREKA electronics installed in the controlled-environment area at $Y-12$. A shielded dete:tor is held in the foreground of the photograph. The terminal for control of the assay in the operations area is shown in front of the hardcopy unit. 
results (uraniun concentrations and inventory) are printed at the end of each analysis.

Figure $4(a)$ is a photograph of the shielded detector assemblies before shipment to $Y-12$. Figure $4(\mathrm{~b})$ is a photograph taken in the $\mathrm{Y}-12$ solution recovery area showing a shielded detector mounted on a stainless steel section of a solvent extraction column.

The most accurate and precise results for uranium concentration can be achieved by measurements of single-phase solutions in the static columns at the normal inventory period when the columns have been shut down and pulsed to disengage phases. However, $17 \%$ of the system inventory resides in the plumbing external to the solvent extraction columns. Each plumbing iine either feeds or is fed by solution in the column. Therefore, column concentration profiles can also be used to deduce uranium concentrations in solutions that reside in the solution feed and drain lines. Measurements to obtain concentration profiles of the static coiumns are performed after shutdown and pulsing of the columns so that the memory of the concentration in the exterral plumbing is effectively erased from the static profiles. However, profiles obtained from measurements of the columns during pulsed operation (just before shutdown for inventory) provide the concentration information necessary to determine the inventory in the external plumbing. Therefore, the columns are measured first during pulsed operation before the monthly shutdown for inventory and again following shutdown and pulsing for phase separation.

The assay system has been designed to automate (under computer control) all operations except for positioning the detectors, performing the appropriate sequence of process operations during the assay, and providing input of process information used by the assay. Control of the assay is accomplished either remotely at the main terminal (in the controlled-environment location) or at a portable terminal in the process area [refer to Figs. 3(b) and $4(a)$ ] so that the process operator can perform the assay. If the assay is controlled at the portable terminal, this equipment can be used for process monitoring.

\section{B. Evaporator Monitor}

Concentration of uranium in solution for feed into the $Y-12$ secondary solvent extraction system is carried out by the secondary intermediate evaporator. This is a recirculating, steam-jacketed unit, $26 \mathrm{~m}$ tall, with an isolated 9-cm-diam stainless steel pipe for solution return to the steam-jacketed 


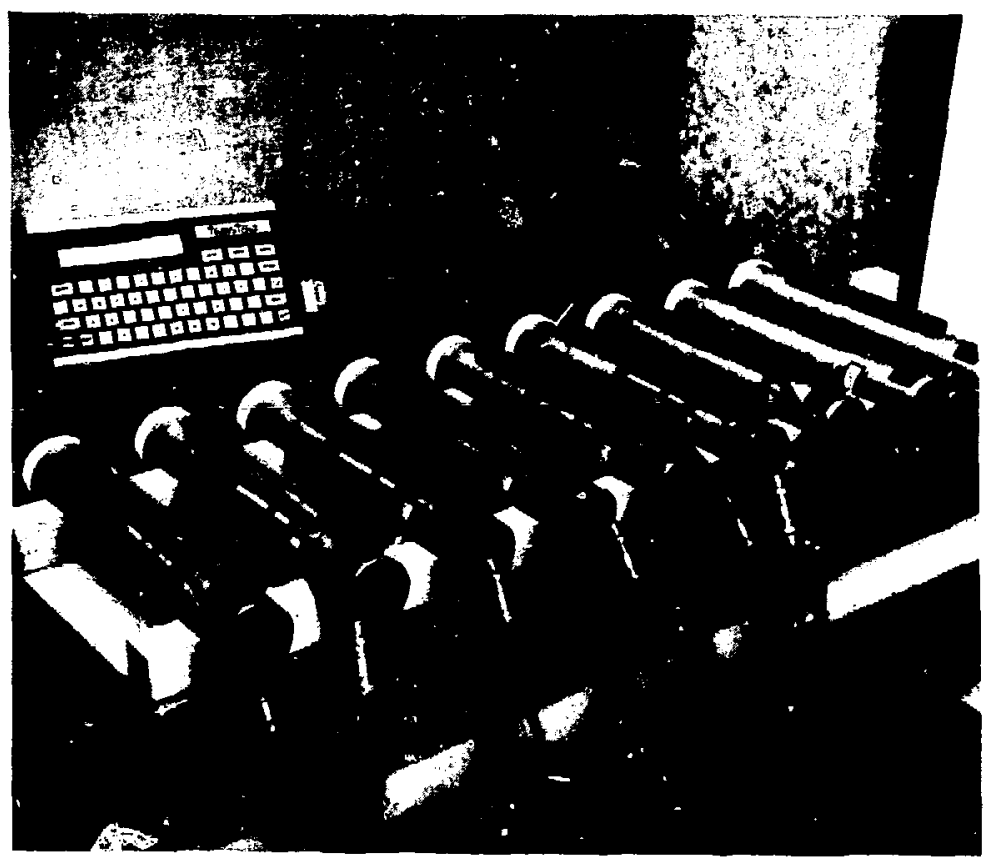

Fig. 4(a). Shielded detectors for EUREKA, assembled before shipment to $Y-12$. The uranium (working standard) disks, mounted on the tungsten holders, are shown inserted in the six left-most collimator shields. The portable terminal for control of the assay in the operations area is in the background.

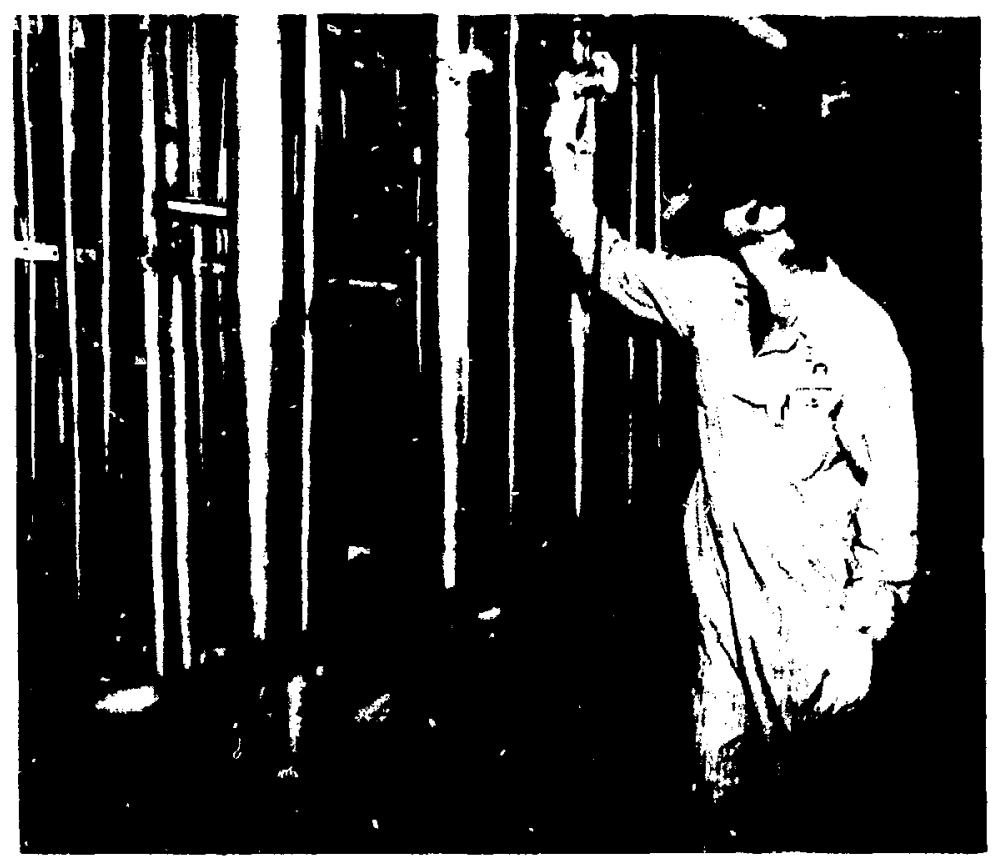

Fig. 4(b). Operator at the Y-12 HEU scrap recovery facility is shown positioning a EUREKA detector on the extraction column of the secondary solvent extraction system. 
section. The evaporator is controlled by manual operation of feed and product flow rates based on monitoring of the specific gravity of solutions withdrawn from the evaporator during operation. This time-consuming process causes frequent upsets in operation. Furthermore, the specific grav$i$ ty is strongly dependent on acid molarity in these highly acidic solutions. To avoid overconcentrating and precipitating uranium and to assure the desired uranium concentration for solvent extraction feed, a shielded NaI(TI) detector was installed on the evaporator return 10op, and an automated instrument was designed to provide a near-real-time readout of uranium concentration in the evaporator solutions.

Figure 5 is a conceptual illustration of the monitor installed on the $Y-12$ secondary intermediate evaporator. The electronics components, located $25 \mathrm{~m}$ from the evaporator, are enclosed in an environmental cabinet through which instrument air flows continuously. The small, programmable

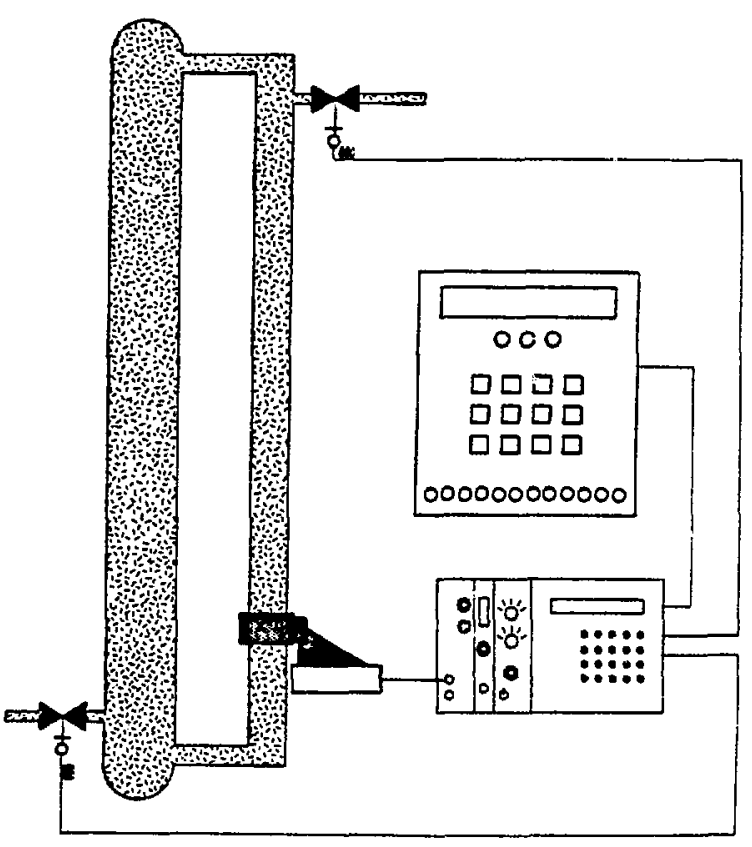

Fig. 5. Conceptual illustration of uranium concentration monitor installed on the $Y-12$ secondary intermediate evaporator. The shielded detector is shown mounted on the evaporator solution return loop. The electronics package (right), located $5 \mathrm{~m}$ from the evaporator, consists of (1 eft to right at the bottom) a stabilized amplifier, high-voltage supply, ADC, and programmable data acquisition and control (PDAC) unit. The LED display unit is also shown. The capability for automatic control of valves by the PDAC unit, al though not currently implemented, is illustrated here.

data acquisition and control (PDAC) unit automates the assay and the monitor readout. The readout panel displays the concentration on a numerical lightemitting diode (LED) display and in an array of colored lights (visible to the operator controlling the evaporator solution flow rates) that indicate concentration range. The readout is updated, typically, at 1-min intervals. The electronics cabinet and the shielded $\mathrm{NaI}(\mathrm{TT})$ detector are shown, installed at $Y-12$, in Figs. $6(a)$ and $6(b)$, respectively. 


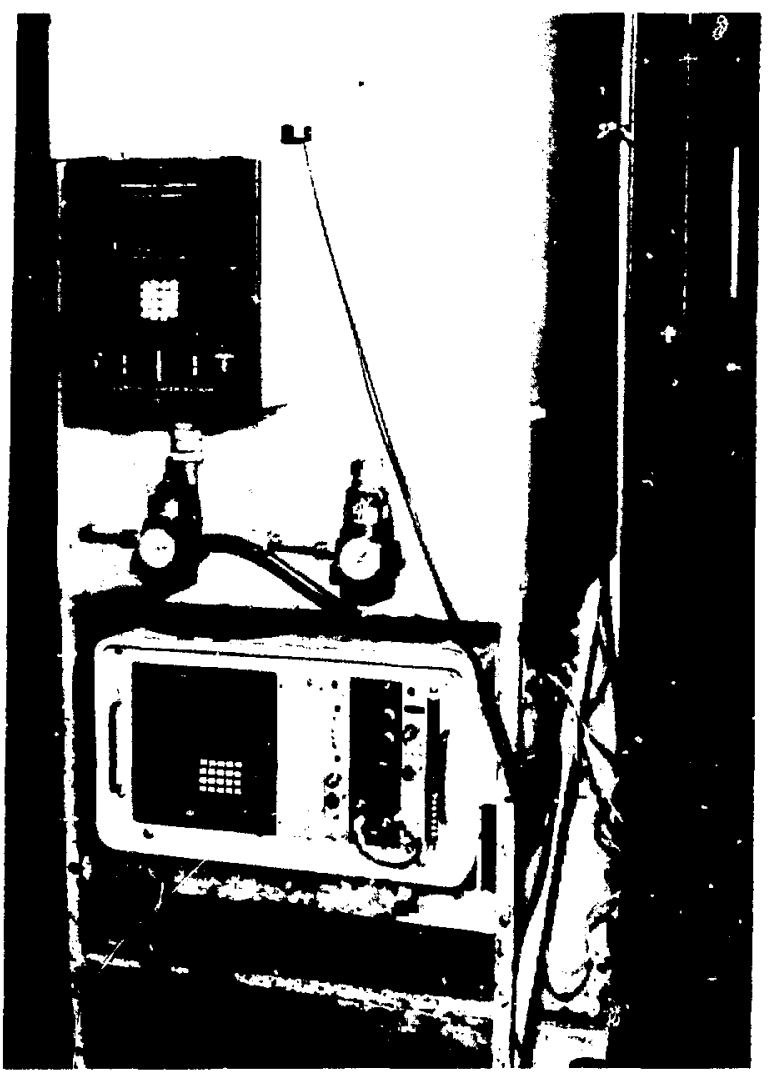

(a)

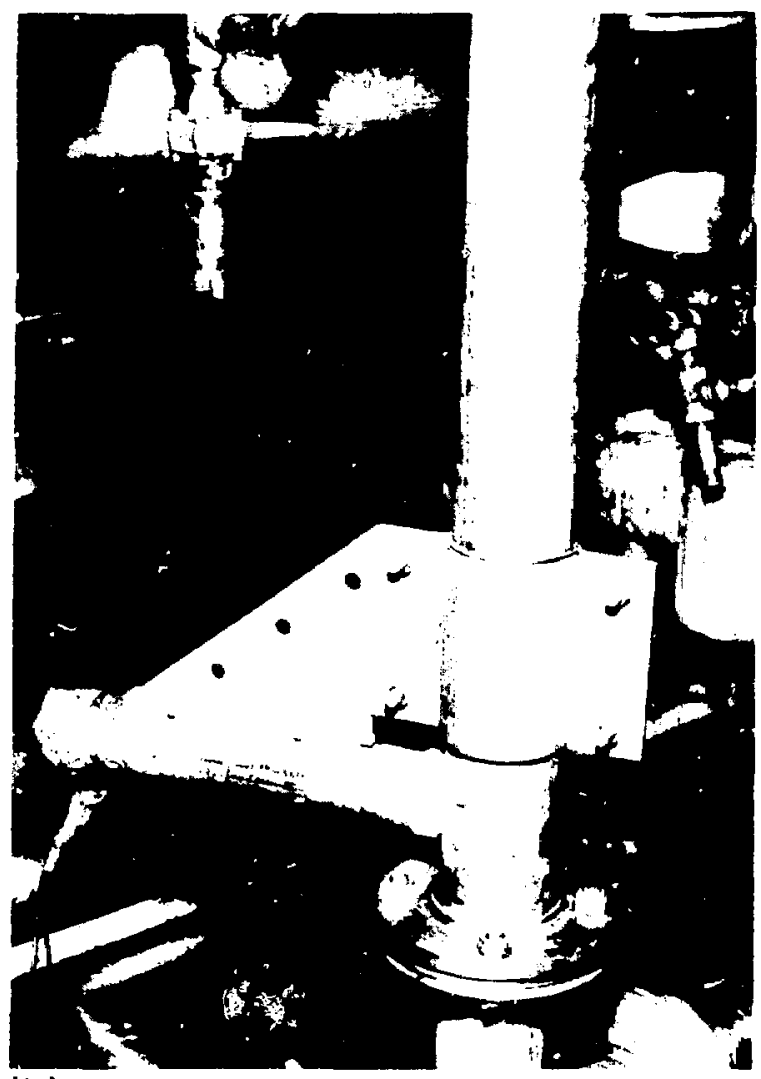

(b)

Fig. 6. The equipment for the uranium concentration monitor for the secondary intermediate evaporator is shown installed at $Y-12$. (a) The electronics components in the environmental enclosure are (1eft to right) the PDAC unit, the high-voltage power supply, the stabilized amplifier, and the ADC. Above the environmental cabinet is the LED display unit. (b) The shielded NaI(TI) detector is mounted on the return loop of the secondary intermediate evaporator.

\section{Uranium Concentration Assay}

The counting geometry for assay of uranium concentration in the solutions in the secondary solvent extraction system is illustrated in drawings of the radial and longitudinal column cross sections, Figs. 7 and 8 , respectively. $A$ similar geometry applies to the assay of solutions in the evaporator return 100p, except that the thickness and inner diameter of the stainless steel pipe are 0.55 and $8.9 \mathrm{~cm}$, respectively, and there are no sieve plates and stator rods to consider.

The assay signature for the uranium concentration measurement is the $186-\mathrm{keV}$ gamma ray of ${ }^{235} \mathrm{U}$. Therefore, the accuracy of the assay relies on a known value of the uranium enrichment in these solutions. 


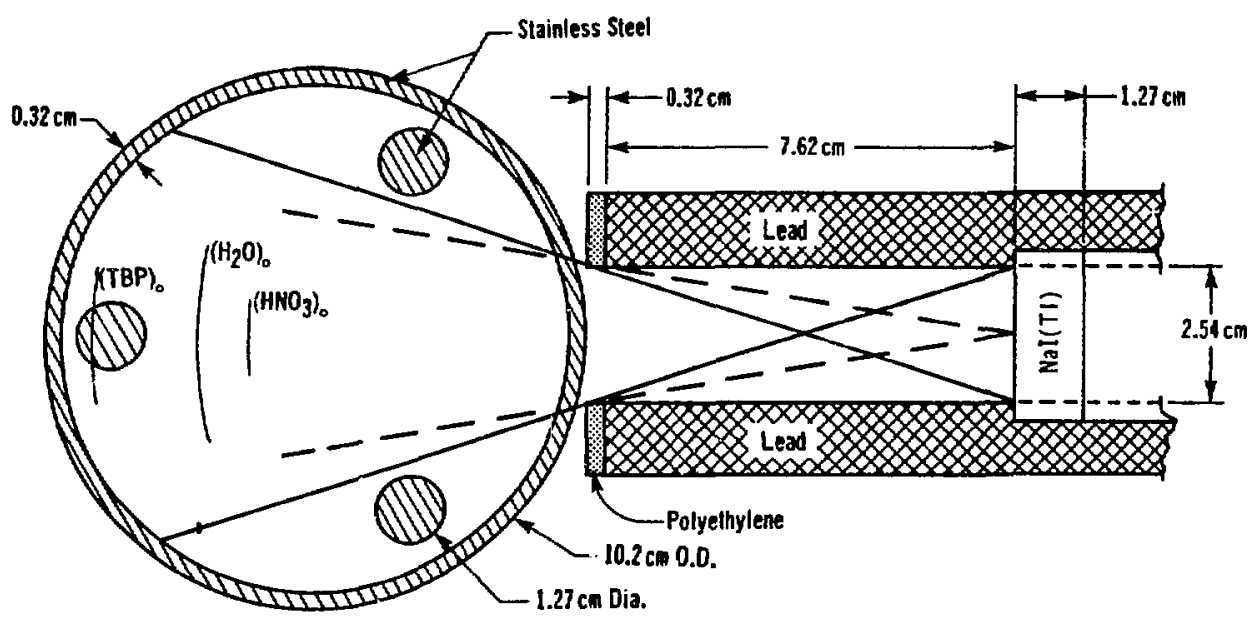

Fig. 7. Drawing (to scale) of a radial cross section of a stainless steel section of a secondary solvent extraction column in the active region. The detector position with respect to the stainless steel stator rods corresponds to the actual positions of the EUREKA detectors on the solvent extraction columns. The curves labeled $\left(\mathrm{H}_{2} \mathrm{O}\right)_{0},\left(\mathrm{HNO}_{3}\right)_{0}$, and (TBP) $)_{0}$ mark one mean free path for 186-keV gemma rays in pure water, nitric acid (4 M), and $30 \%$ tributyl phosphate in kerosene.

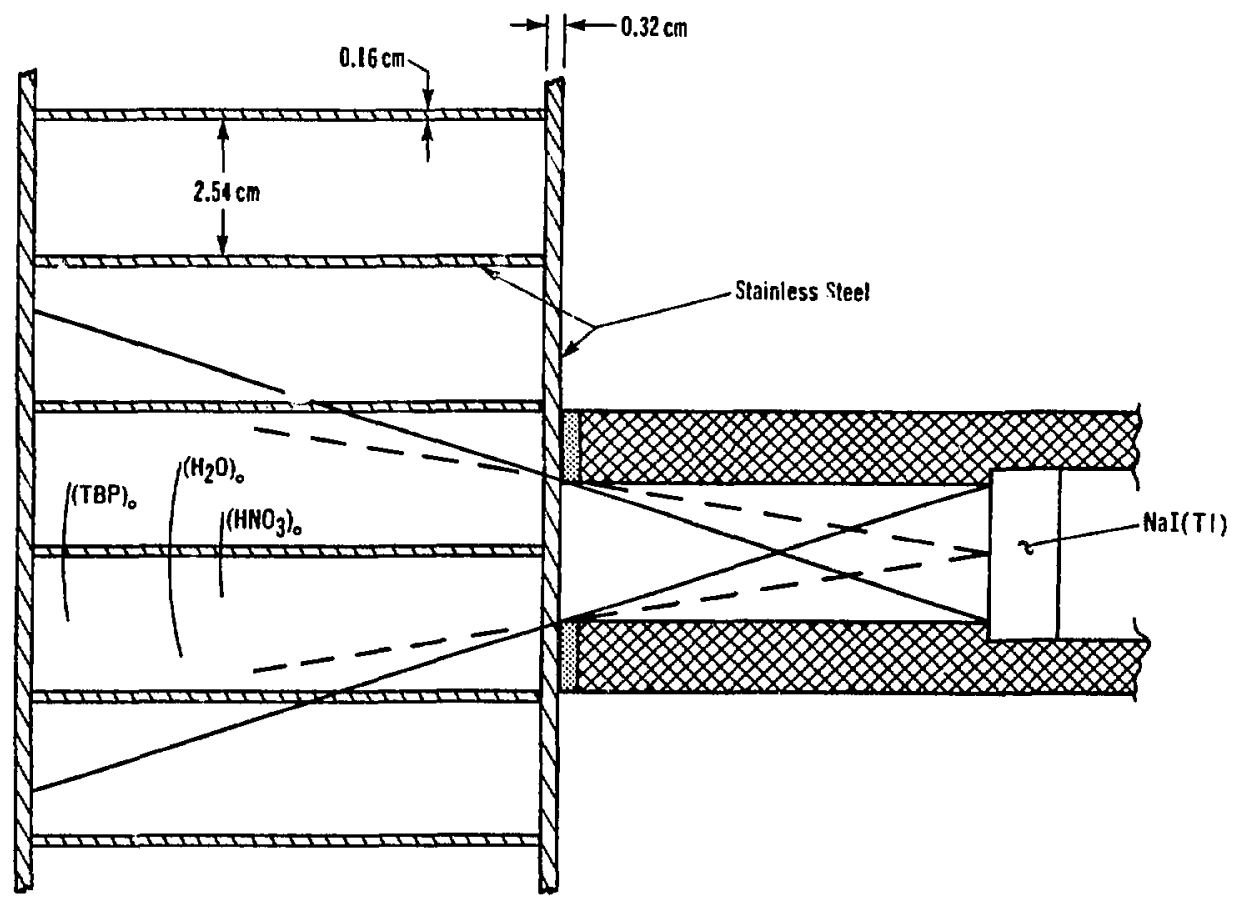

Fig. 8. Drawing (to scale) of a longitudinal cross section of a stainless steel section of a secondary solvent extraction column in the active region. The detector position with respect to the sieve plates corresponds to the maximum count-rate configuration for $186-\mathrm{keV}$ gamma rays. Refer to Fig. 7 caption for definitions. 
Because solvent densities in the secondary solvent extraction system can vary by $25 \%$ about unity, solution self-attenuation effects are solvent dependent. Thus, calibrations were calculated for each solvent category. The calculation for the low-acid, aqueous solutions within the stainless steel portions of the solvent extraction columns was verified empirically.

The calculation integrates the $186-\mathrm{keV}$ activity (at the cone apex) from a truncated right-circular cone-shaped source of uniform activity. It includes the attenuation effecis of the sample and external absorbers and incorporates a reduced scattering attenuation to account for gamma rays that scatter but are still detected within the resolution limits of the 186-keV photopeak. The calculation does not include the effects of the sieve plates and stator rods.

Fabrication of a stainless steel solution cell (Fig. 9) was required to reproduce the construction and configuration of the $Y-12$ secondary soivent extraction columns for verification of the calculation. The lower half of the $50-\mathrm{cm}-1$ ong by $10-\mathrm{cm}-$ diam cell contained appropriately positioned plates and rods designed to match the sieve plates and stator rods that are in the active regions of the solvent extraction columns. Separate measurements were performed with the reference detector positioned on the upper and the lower half of the cell. To el iminate the effects of count-rate losses on the assay result, the measured 186-keV count rate was normalized to that for the $60-\mathrm{keV}$ photopeak produced by a $0.5-\mu \mathrm{Ci}{ }^{241}$ Am source attached to the detector in fixed geometry. (The normalization factor differs for each detector primarily because of differences in the ${ }^{241} \mathrm{Am}$ source strengths.) Measurements with

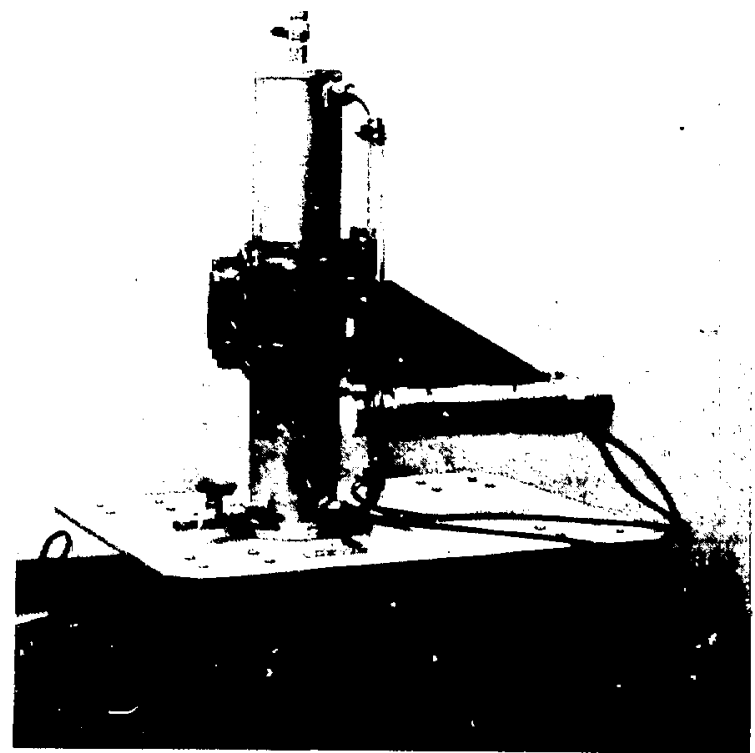

Fig. 9. Stainless steel sample cell designed to reproduce the construction and internal configuration of the stainless steel sections of the $\mathrm{Y}-12$ secondary solvent extraction columns. The shielded detector is shown mounted on the lower half of the cell, which is fitted internally with stator rods and sieve plates. 
the reference detector were rerformed using five well-characterized HEU solutions with uranium concentrations of $7,59,129,178$, and $258 \mathrm{~g} / \mathrm{l}$.

The absolute 186-keV count rate measured with the reference detector positioned on the upper half of the cell is within $1 \%$ of the calculated value for each reference solution with a sample standard deviation of $0.5 \%$ in the ratio (calculation to reference value). The corresponding 186-keV count rates (corrected for count-rate losses by the 60-keV rate) and the calculated calibration normalized to these results are shown in the upper portion of Fig. 10 by the large dots and the solid line, respectively. The ratios of the measured to the calculated values are shown as solid points in the lower portion of Fig. 10. The relative standard deviation in this ratio for the five samples is $0.7 \%$.

The count rates measured with the reference detector positioned on the lower half of the cell are also plotted in Fig. 10. These include results

Fig. 10. The corrected ret 186-keV photopeak areas (the 186-keV area corrected for count-rate 10 sses by $60-\mathrm{keV}$ area) measured with the reference detector mounted in different locations on the stanuard stainless steel sample cell. Measurements were performed with five different reference solutions of HEU (top). The solid curve is the calculation that applies to the measurements in the inactive (no plates) region (large dots). The ratios of these points to the calculated curve are shown at the bottom. A measurement control data point obtained during each set of solution assays with the reference uranium disk inserted in the collimator is also plotted (asterisks). Measurements in the active region are indicated by crosses (plates only) and triangles (plates and rods).

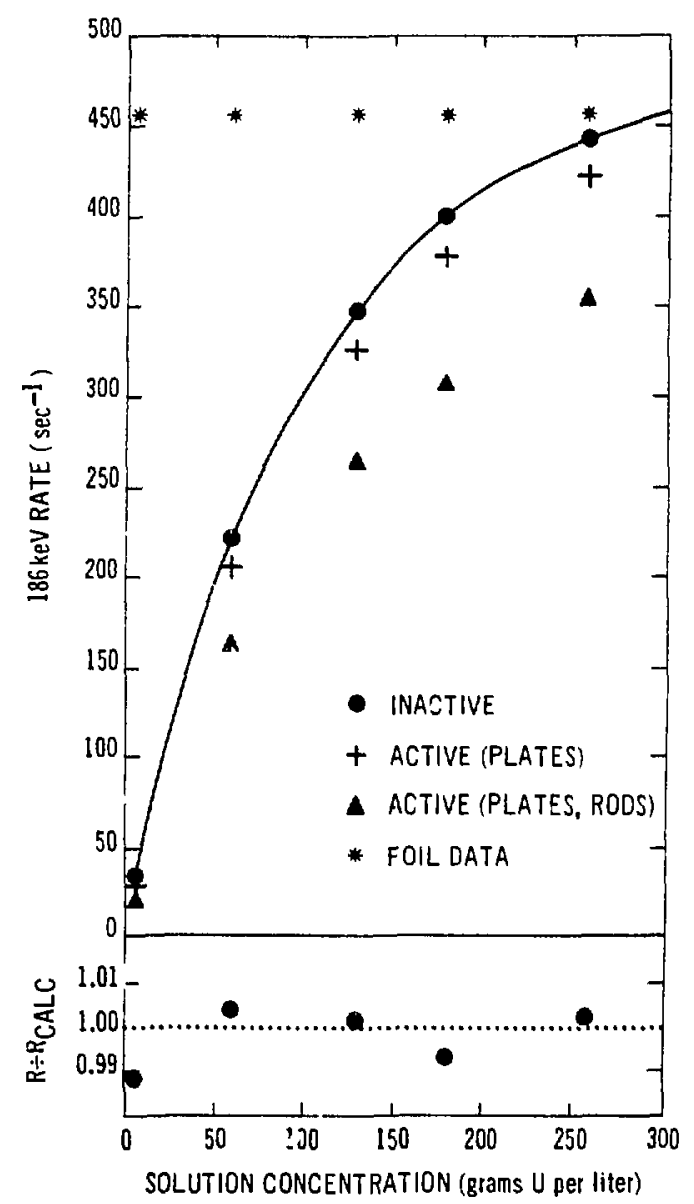


obtained with the detector positioned between two rods (crosses) as illustrated in Fig. 7 and results obtained with the detector positioned directly in front of a rod (triangles). The measured effects (relative to results obtained on the upper portion of the cell) of the sieve plates are plotted in Fig. 11 as the 186-keV ratio vs uranium concentration. The measured effects of the stator rods are plotted in Fig. 12.

The large reductions in the $186-\mathrm{keV}$ count rate $(20 \%$ or more) caused by the stator rods were avoided in the EUREKA assays by positioning the detectors between the rods on the active regions of the solvent extraction columns. The smaller effects of the sieve plates, as represented by the quadratic fit to the data in Fig. 11, were incorporated in the EUREKA assays of solutions in the active portions of the columns by applying this empirical correction to the measured count rates in an iterative manner. The error bars on the data in Fig. 11 indicate the high and low results obtained from measurements with the collimator centered on a plate and hetween two plates, respectively. The collimated EUREKA detectors are randomly positioned on the active portions of the

Fig. 11. The effect of the sieve plates on the 186-keV count rate is illustrated by the data points, which are the ratios of the crosses to the large-dot data points of Fig. 10, plotted vs reference solution concentration. The actual data points shown are each an average of two results: one with the detector collimator positioned as in Fig. 8 and one with the detector collimator positioned between two sieve plates. The error bar limits (upper and lower) show these two results, respectively.

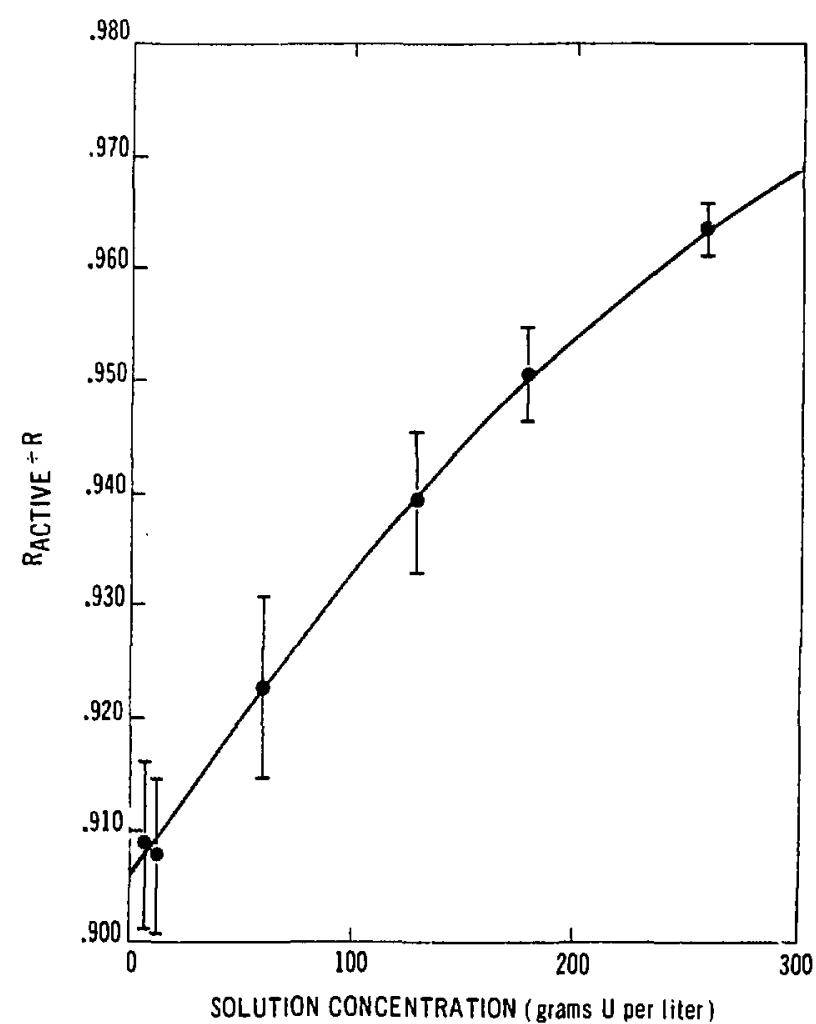




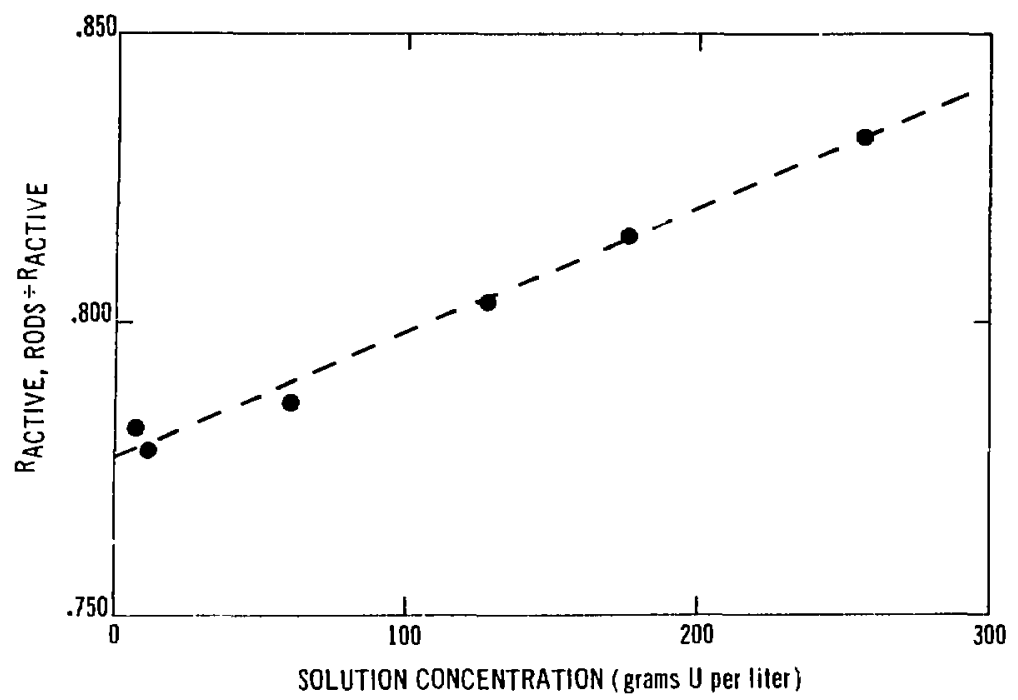

Fig. 12. The 1arge effect of a stator rod, positioned directly in front of the detector, on the $186-\mathrm{keV}$ count rate is illustrated by the data points, which are the ratios of the triangular to the cross data points of Fig. 10, plotted vs reference solution concentration. EUREKA avoids these effects by positioning detectors with respect to rods as shown in Fig. 7 .

column relative to the plate positions. This introduces a small uncertainty into the concentration assay.

A separate calculation of count rate vs uranium concentration was performed for each column dimension (including that for the evaporator return $100 p)$ and for each solvent phase. A specific, representative acid molarity was assumed for each calculation. The inverse of the calculated results (concentration vs count rate) was fitted with a ninth-order polynomial, and the fit parameters were stored in the data acquisition programs. EUREKA corrects - the measured count rates for solutions with acid molarities different from those assumed in the calculation in a second iteration in solving for concentration. The correction factor, CF, is

$$
C F=M \cdot\left(0.005-0.009 p_{u}\right)+1,
$$


where $M$ is acid molarity and $p_{u}$ is uranium concentration in grams per milliliter. This correction is derived from fits to the numerical integration results applied over the useful range of acid molarity.

A polynomial fit to the numerical integration results was the simplest approach to the calibration for several reasons: (1) double precision arithmetic to obtain the necessary sensitivity in the sriginal numerical integration is avoided, (2) an inverse form of the numerica integration was not apparent, and (3) the ninth-order polynomial fit gives better than $0.2 \%$ agreement over $90 \%$ of the concentration range and better than $0.5 \%$ in the 1 owest $10 \%$ of the range.

Several random effects ran be quantified to give an estimate of the uncertainty in the EUREKA concentration assay results. These include the effects of counting statistics, the effects of the position of the collimators with respect to the sieve plates, and the effects of the uncertain knowledge of acid molarity. The sieve plate effects are not truly random because a given detector holder, once positioned, will remain fixed relative to the plates. However, averaged over many detector positions where concentrations vary, the effect on the concentration result can be treated as random.

Table I shows the random uncertainties in the measured $186-\mathrm{keV}$ count rates due to counting statistics (in 600-s counts) and positioning (relative to the sieve plates) for three concentrations spanning the assay range. The net result in Table I is the quadratic sum of these two uncertainties. Table II

TABL.E I

RELATIVE RANDUM UNCERTAINTY (10) IN THE 186-KEV COUNT RATE DUE TO COLNTING STATISTICS AND SIEVE PLATES

\begin{tabular}{rccc}
$\rho_{\Perp}(G / 2)$ & $T=600 \mathrm{~S}$ & PLATES & NE T \\
\hline 10 & $1.1 \mathrm{X}$ & $0.8 \mathrm{X}$ & $1.4 \mathrm{X}$ \\
125 & $0.4 \mathrm{X}$ & $0.6 \mathrm{X}$ & $0.7 \mathrm{X}$ \\
250 & $0.3 \mathrm{X}$ & $0.2 \mathrm{X}$ & $0.4 \mathrm{X}$
\end{tabular}

shows the random uncertainties in the measured $186-\mathrm{keV}$ count rates due to uncertain knowledge of solution acid molarities for the same concentrations. These results are shown separately for measurements of the static and pulsed columns because the uncertainties are significantly larger for the latter. 
RELATIVE RANDGM UNCERTAINTY (10) IN THE 186-KEV COUNT RATE DUE TO UNICERTAIN KNOWLEDGE OF ACID MOLARITY*

\begin{tabular}{|c|c|c|c|c|}
\hline \multirow[b]{2}{*}{$\rho_{U}(G / R)$} & \multicolumn{2}{|c|}{ HIGH ACIO } & \multicolumn{2}{|c|}{ LOW ACID } \\
\hline & PULSED & STAIIC & PULSED & $\overline{\text { STATIC }}$ \\
\hline 10 & $2.8 x$ & $0.8 x$ & $1.5 x$ & $0.6 x$ \\
\hline 125 & $2.2 \%$ & $0.6 \%$ & $1.1 \%$ & $0.5 x$ \\
\hline 250 & $1.7 \%$ & $0.5 \%$ & $0.9 x$ & $0.4 x$ \\
\hline
\end{tabular}

"ASSUMES KNOWLEDGE OF HIGH-ACID SPECIFIC GRAVITY TO $\pm 3 \%$ (THAT IS, ACID MOLARITY IS KNOWN TO $\pm 20 \%$ ). ASSUMES KHOWLEDGE OF LOW-ACID SPECIFIC GRAYITY TO $22 \%$ (THAT IS, ACID MOLARITY IAAN VARY BETWEEN 0 AND 2).

" ASSUMES UNCERTAINTY (2o) IN THE AVERAGE SPECIFIC GRAVITY OF MIATR IX TO BE $1 / 2$ THE DIFFERENCE IN SPECIFIC GRAVITY OF THE AQUECIUS AND ORGANIC PHASES.

Table III shows the quadratic sums of the net results from Table I and the results from Table II.

Because of the increasing self-attenuation of the 186-kel count rate with increasing uranium concentration, the relative uncertainty in uranium concentration is much larger than the relative uncertainty in count rate at high uranium concentrations. The differential relative uncertainties for the three representative concentrations are given in Table IV. The product of this quantity and the net random uncertainty in the $186-\mathrm{keV}$ count rate (Table III) gives the net random uncertainty in the measured uranium concentration. These results are given in Table $V$.

TABLE III

NET RELATIVE RANDON UNCERTAINTY (10) IN 186-KEV COUNT RATE

\begin{tabular}{|c|c|c|c|c|}
\hline \multirow[b]{2}{*}{$\rho_{U}(G / R)$} & \multicolumn{2}{|c|}{$\mathrm{HIGH} A C I D$} & \multicolumn{2}{|c|}{ LOW ACID } \\
\hline & PULSED & SIATIC & PULSED" & $\overline{\text { STATIC }}$ \\
\hline 10 & $3.1 \%$ & $1.6 \%$ & $2,1 x$ & $1.5 x$ \\
\hline 125 & $2.3 \%$ & $0.9 x$ & $1.3 x$ & $0.9 x$ \\
\hline 250 & $1.8 \%$ & $0.7 x$ & $1.0 x$ & $0.6 x$ \\
\hline
\end{tabular}

"ASSUMES UNCERTAINTY (20) IN THE AVERAGE SPECIFIC GRAVITY OF MATRIX TO BE 1/2 THE DIFFEREHCE IN SPECIFIC GRAVITY OF THE AQUEOUS AND ORGANIC PHASES.

TABLE IV

DIFFERENTIAL RELATIVE UNCERTAINTY IN URANIUM CONCENTRATION VERSUS THAT IN COUNT RATE

$\begin{array}{cc}\rho_{U}(G / R) & {\left[\delta \rho_{U}(x)\right] /\left[\delta N_{186}(x)\right]} \\ 10 & 1.06 \\ 125 & 2.03 \\ 250 & 4.08\end{array}$


TABLE V

RELATIVE RANDOM UNCERTAINTY (10) IN MEASURED URANIUM CONCENTRATION

\begin{tabular}{|c|c|c|c|c|}
\hline \multirow[b]{2}{*}{$D_{1}(G / 2)$} & \multicolumn{2}{|c|}{ EXIRACIION. SIRIP } & \multicolumn{2}{|c|}{ BACKWASH } \\
\hline & PULSED* & STAIIC & PULSED" & STAIIC \\
\hline 10 & $3.3 x$ & $1.7 x$ & $2.2 x$ & $1.6 x$ \\
\hline 125 & $4.7 x$ & $1.8 x$ & $2.6 x$ & $1.8 \mathrm{x}$ \\
\hline 250 & $7.3 x$ & $2.9 \mathrm{x}$ & $4.1 \%$ & $2.4 x$ \\
\hline
\end{tabular}

- ASSUMES UNCERTAINTY (20) IN THE AVERAGE SPECIFIC GRAVITY OF MA-

TRIX TO BE $1 / 2$ THE DIFFERENCE IN SPECIFIC GRAVITY OF THE AQUEOUS

AND ORGAHIC PHASES.

The data in Table $V$ can be used to predict the minimum random uncertainty in the EUREKA inventory. For simplicity, it is assumed that each of the six EUREKA detectors assays a uranium concentration of $100 \mathrm{~g} / \ell$. The total volume of solution in a column is approximately $60 \ell$. Thus, the corresponding uncertainty in the $6-\mathrm{kg}$ column inventory is approximately $1 \%$ for a static column and $2 \%$ for a pulsed column if there are no other random effects.

D. Measurement Control

Measurement control applied to these in-line NDA instruments includes: systematic monitoring of photopeak centroids, photopeak widths, and count rates of all spectra acquired; regular measurements of backgrounds; and regular assays of (working "standard") enriched uranium disks. The accepted values for photopeak widths and centroids are stored in parameter files accessed by the EUREKA and evaporator monitor codes. These values are compared with the measured values. The data are automatically flagged if the deviation exceeds the prescribed value. Because analog spectrum gain stabilization is employed and because count rates are 10w ( $3000 \mathrm{~s}^{-1}$ ) and relatively constant (the ${ }^{241}$ Am is responsible for most of the rate), these flags are rarely raised. Background and foil measurements are performed at intervals prescribed by the users. The schedules differ for the two instruments, but the methods are the same, and the results of the background and foil measurements have the same significance for both instruments.

The method for background measurement involves insertion of a 5-mm-thick tungsten shield into a slot near the end of the collimator shield, followed by a normal assay for determination of the ratio of net peak areas (of the 186and 60-keV photopeaks). These results are subtracted from subsequent assays 
of samples. Backgrounds are normally stored in the parameter file by the evaporator monitor code. This effort is completely automated (through execution of a separate background assay option) by the EUREKA code, which stores a separate background result for each detector mounted on each of the three columns. (A total of 18 EUREKA background results are stored.) These stored values are updated each time backgrounds are remeasured or background spectra are read into memory from floppy $d_{1}$, kette files. Background ratios of net peak areas are typically 1 to $5 \%$ of the ratio measured in a solution assay for which the uranium concentration is $100 \mathrm{~g} / \mathrm{h}$.

Assay of uranium disks for measurement control involves insertion of tungsten-backed 13-mm-diam disks of HEU metal (total weight is $3 \mathrm{~g}$ per disk) into the same slots in the collimator used for the background shields [Figs. 3(b) and $4(a)]$. The uranium disks are mounted on one end of the tungsten background shields. Each detector has its own unique disk. The disk position is reproducible in that the uncertainty in the 186-keV count rate because of positioning is less than the random uncertainity from counting statistics. The ratio of net peak areas determined in the disk assay is compared (automatically in the EUREKA code) with the nominal values for each detector. A change in the efficiency factor affects the measured foil ratio proportionally. Therefore, the foil result has been used to monitor and correct the efficiency factor for each detector.

The efficiency factor is defined as a relative counting efficiency (proportional to the ratio of efficiencies for counting the net peak areas of the 186- and 60-keV photopeaks) for a given detector relative to that for the reference detector. This quantity is stored by each detector in the EUREKA parameter file. It can be measured directly by assay of a reference solution of uranium in the standard cell, or the directly measured values can be corrected by the changes in the disk assay results. The latter method has proved to be a simple, accurate, and essential alternative to the time-consuming effort of direct measurements of solutions in the standard cell.

The average relative standard deviation in the six disk assay results ottained (before each static and pulsed assay on each of the three columns) during inventories since February 1983 is $0.35 \%$ for all six detectors. This is only slightly larger than the contribution of counting statistics $(0.3 \%)$ to the random uncertainty. Therefore, any effects of the location on the column, the column identity (extraction, strip, or backwash), or the column operational 
status (pulsed or static), as well as effects cf disk positioning in the shields, are small enough to be ignored in using the disk assay results to correct the detector efficiency parameters.

E. Results and Significance of Measurement Control by Uranium Disk Assay

Measurement control by disk or foil assay has been demonstrated as a method to monitor the normalization of the calibration of solution assay instruments that use methods of high-resolution gamma-ray measurement based on both passive gamma-ray assay and on gamma- or x-ray transmission measurements. 6 The greater overall stability in the high-resolution systems reduces the required frequency of direct measurement of the calibration normalization (CN) even though the desired accuracy greatly exceeds that achievable by NaI(TI) detectors. Therefore, foil assay results have been used in the past to monitor the $\mathrm{CN}$ of a high-resolution gamma-ray instrument on a frequent basis. Adjustment of the high-resolution system $\mathrm{CN}$, which is required only infrequently, is accomplished directly using well-characterized reference solutions.

It is not practical to rely on the availability of reference solutions to satisfy the frequent needs for normalization of the calibrations of NaI(TI) assay instruments. Furthermore, the time and effort required to apply such normalization methods frequently to multiple detectors designed to operate on1 ine are prohibitive. Therefore, the $Y-12$ instruments have relied on the disk assay results to provide the necessary normalization updates. The extensive disk assay data obtained with the EUREKA detectors (a disk assay has preceded each concentration assay performed on the solvent extraction columns to date) illustrate the effectiveness of these methods.

Figure 13 is a plot of the efficiency factors (or CNs) deduced from the disk assay results $y$ s date for each of the six EUREKA detectors during the first year of operation at $Y-12$. With two exceptions, each (solid) data point since the January measurements is the average of six 600-s disk measurements. (These are the disk assays that precede the pulsed and static solution assays on each of the three columns during the monthly measurements for inventory.) The exceptions are two sets of disk measurements performed in mid-May and in mid-September, concurrent with direct measurements at $Y-12$ of the CNs (open points) for each detector using a well-characterized reference solution and the calibration cell. The dashed line indicates the CN measured for each detector by direct methods during the original calibration at Los Alamos. During this 

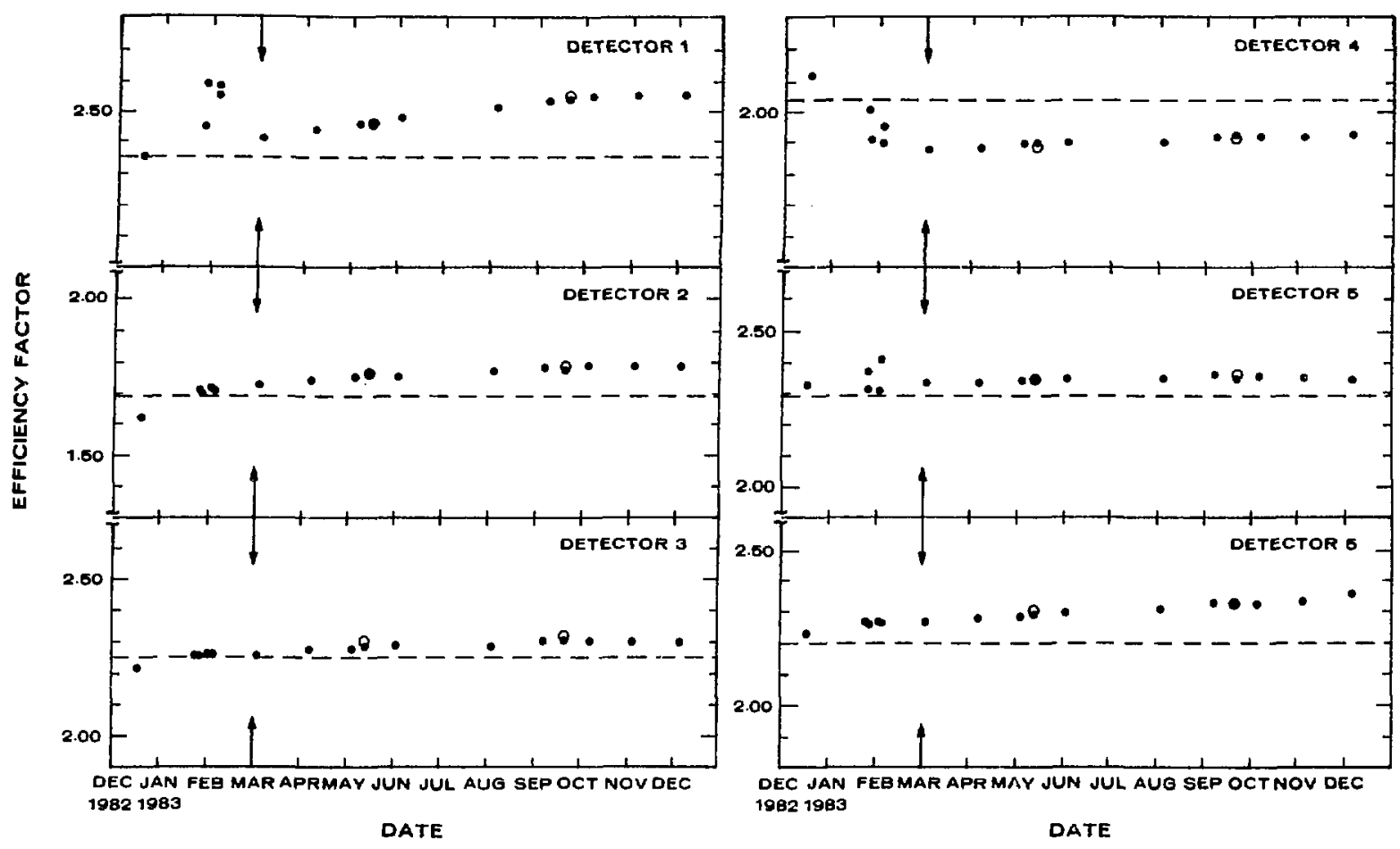

Fig. 13. The efficiency factor (or $\mathrm{CN}$ ) deduced from disk assays (solid points) vs date is plotted for the six EUREKA detectors. With the exception of the points plotted at mid-May and at mid-September, each point since January 1983 corresponds to the average of six disk measurements obtained before solution assays on each of the three columns in the pulsed and static modes during inventory. The dashed lines correspond to the CNs determined by direct measurements, performed in November 1982 at LOS Alamos, of reference solutions in tine standard cell. The open circles plotted at mid-May and at mid-September are direct measurements performed at $\mathrm{Y}-12$ using the standard cell and a uranium reference solution. Disk measurements, which were also performed at this time, are plotted as well. The vertical arrows mark the date on which the detector positions were fixed in the shields.

original calibration, the characteristic (186- to 60-keV) ratios, which have been used for comparison with all successive disk assays, were precisely determined.

The initial measurements with detector 4 just after installation of EUREKA indicated the possibility of electronic malfunction in that detector. It was subsequently disassembled, checked, and reassembled in its shield. In this process, the ${ }^{241}$ Am source geometry was changed, causing a large $(10 \%)$ decrease in the $\mathrm{CN}$. The availability of the well-characterized uranium disk made 
possible the immediate use of this detector after reassembly, without the need to normalize directly using a reference solution and the standard cell.

Large fluctuations in the CNs deduced from disk assays during December through February indicated a possible shifting (sliding) of several of the $\mathrm{NaI}(\mathrm{Tl})$ detectors in the shields. Just before the March inventory, nylon set screws were installed to fix the proper detector positions. Since that time, only small, gradual monotonic changes in the CNs were observed.

The direct calibration measurements performed in mid-May with a $100-\mathrm{g} / \mathrm{l}$ uranium reference solution in the standard cell produced CNs in excellent agreement with the disk predictions (Fig. 13). The average deviation of the disk prediction from the direct measurement was $0.4 \%$ and the standard deviation was $0.5 \%(10)$. This is an indication of the accuracy with which the disk result tracks the $\mathrm{CN}$. Similar agreement was observed for the results of the September direct calibration measurements.

Table VI is a tabulation of data on EUREKA detector CNs deduced from the disk results. The quantity $\mathrm{ACN}$ is the average $\mathrm{CN}$ deduced from 5 months (centered in mid-May 1983) of disk data acquired since early March 1983 when the detector positions in the shields became fixed. The standard deviation (10) in the (solid) data points plotted in Fig. 13 during this period is also given. The per cent deviation between $A C N$ and the original $C N\left(C N_{0}\right)$ derived during the direct calibration at Los Alamos is tabulated for each detector. This latter quantity is a measure of the relative magnitude of the drift in the relative detection efficiency between the time the $\mathrm{CN}_{0}$ values were measured and mid-May 1983 ( 26 months). Except for detector 4, for which this shift was anomalously large and negative $(-7.5 \%)$, because of mispositioning the ${ }^{241} \mathrm{Am}$

TABLE VI

EUREKA DETECTOR EFFICIENCY FACTOR

DATA DEDICED FROM FOIL ASSAYS

\begin{tabular}{|c|c|c|c|}
\hline DETECTOR ID & $\mathrm{ACN}$ & 10 & $\frac{\mathrm{ACN}-\mathrm{CN}_{0}}{\mathrm{CN}_{0}}$ \\
\hline 1 & 2.460 & $1.3 x$ & $4.7 x$ \\
\hline 2 & 1.750 & $0.8 x$ & $3.7 x$ \\
\hline 3 & 2.275 & $0.5 x$ & $1.2 x$ \\
\hline 4 & 1.886 & $0.4 x$ & $-7.5 x$ \\
\hline 5 & 2.338 & $0.2 x$ & $2.5 x$ \\
\hline 6 & 2.288 & $0.6 x$ & $3.8 \%$ \\
\hline
\end{tabular}


source after reassembling the detector in the shield, the shift for the 6-month period varies between 1 and 5\%. However, a 5\% bias in the count rate propagates to $10 \%$ in the concentration assay for uranium concentrations of $100 \mathrm{~g} / \mathrm{l}$ (Table IV) and to $20 \%$ at $250 \mathrm{~g} / \mathrm{l}$. The gradual shifts are monotonic and positive for all detectors. Therefore, the corrections applied to the detector CNs have been essential in avoiding a large and increasing negative bias in the inventory results as a function of time.

The cause of the apparent decrease in the relative (186- to 60-keV) net photopeak counting efficiencies is not defined, although it is likely to be electronic. However, because the effect is smooth and gradual with time and because the disk assays have proved to be reliable within the predicted random certainties, the disk measurements can be performed much less often for measurement control purposes.

\section{RESULTS OF SOLVENT EXTRACTION SYSTEM INVENTORY MEASUREMENTS}

A. Introduction

Evaluation of the EUREKA inventory measurement capability included performing the pulsed and static EUREKA assay sequences on the solvent extraction system as described previously, followed by dumping the solution components of the solvent extraction system including external plumbing. The dumped components were analyzed externally for direct determination of solution volumes and uranium concentrations to give uranium masses. These direct results are the reference values used for comparison with the EUREKA inventory results.

EUREKA inventories were performed in 1983 during the first week of each month excluding January, June, and July. The corresponding direct measurements were performed for comparison following five of the EUREKA inventories. The results are presented in detail for those months when both the EUREKA measurements and the direct measurements were performed.

\section{B. Direct Inventory Resul ts}

Aftar making the EUREKA static measurements of uranium concentration in the separated phases in the isolated solvent extraction columns, the solution components of the solvent extraction system were dumped and analyzed. Through 
April 1983, the column dumps included solution volumes in the pulser line. As of May, the pulser volumes were dumped and analyzed as separate components of the external plumbing. The volumes of the columns were drained (from the bottom) into six or seven $10-\ell$ bottles, and each bottle was weighed, homogenized, and sampled. The external plumbing components were handled similarly. The samples were analyzed for specific gravity (by a commercial, ultrasonic device) and for uranium concentration (by nondestructive, high-resolution gamma-ray assay ${ }^{2,7}$ ). Total volumes, determined from the measured weights and specific gravities, were used with uranium concentrations to determine the reference masses of uranium. The estimated random relative uncertainty in the uranium concentration results is $0.7 \%(1 \sigma)$. The estimated uncertainty in volume is $1 \%$ because of weighing. Therefore, the minimum overall random uncertainty in the reference results for each column is estimated to be $0.7 \%$ for uranium mass and $0.4 \%$ for solution volume. The magnitudes of other possible random effects in the draining and sampling processes are unknown.

During July 1982 before installing EUREKA, the procedures for the dumping and external analysis of solvent extraction system solution components were tested at $\mathrm{Y}-12$. After the isolated solvent extraction columns (including the pulser lines) were drained for these tests, the columns were filled with a $50 \%$ $\mathrm{HNO}_{3}$ solution. This flush solution was then drained and analyzed for uranium. Table VII summarizes the column inventory results of this pilot effort. The residual uranium, the quantity of dissolvable uranium that remains in the column after the solution contents are drained, is $1.3 \%$ of the columi inventory. The subsequent direct inventory measurements did not include a flushing procedure, but it is assumed, for the purposes of comparison with EUREKA assays, that $1.3 \%$ must be added to the measured volumes and uranium masses of the dumped components to obtain the reference values.

TABLE VII

URANIUM INVENTORY HELD UP IN SOLVENT EXTRACTION COLUMNS FOLLOWING COLUMN DUAPS

\begin{tabular}{lccc} 
COLUMN & $\begin{array}{c}\text { COLUMN } \\
\text { INVENTORY } \\
\text { IG UI) }\end{array}$ & $\begin{array}{c}\text { COLUMN } \\
\text { HOLDUP } \\
(6 U 1)\end{array}$ & $\frac{\text { HOLDUP }}{\text { INYENTORY }} \cdot 100$ \\
\hline EXTR. & 5655 & 73.4 & $1.30 \%$ \\
STRIP & 8778 & 120.5 & 1.378 \\
BACKW. & 3132 & 39.2 & $1.25 \%$ \\
TOTAL & 17565 & 233.1 & $1.33 \%$
\end{tabular}


Tables VIII and IX give the reference values for solution volumes and uranium masses, respectively, determined directly by measurements of components of the solvent extraction system dumps. The dates given are those of the EUREKA measurements, or one working day before the dump. The results in Tables VIII and IX incorporate the $1.3 \%$ adjustments for residual solution in the columns.

C. EUREKA Inventory Results

The 600-s background, foil, and solution spectra for each of the six EUREKA detectors were acquired in separate 500-channel portions of MCA memory. Figure 14 shows the full background, foil, and aqueous solution spectra (a, b, and $c$, respectively) for detector 5, obtained during the November 1983 static

TABLE VIII

DUMP VOLUMES (MILLILITERS)

\begin{tabular}{|c|c|c|c|c|c|}
\hline \multirow[b]{2}{*}{ DAIE } & \multirow{2}{*}{ COLUMN } & \multicolumn{4}{|c|}{ COMPONENI } \\
\hline & & Colum & PULSER & PLUMBIMG & TOIAL \\
\hline \multirow[t]{4}{*}{$2 / 4 / 83 *$} & EXTR. & \multicolumn{2}{|c|}{64672} & 20064 & 84736 \\
\hline & STRIP & \multicolumn{2}{|c|}{63651} & 13725 & 77376 \\
\hline & BACKW. & \multicolumn{2}{|c|}{.63438} & 8396 & 71834 \\
\hline & TOTAL & \multicolumn{2}{|c|}{191761} & 42185 & 233946 \\
\hline \multirow[t]{4}{*}{$3 / 4 / 83^{\prime \prime}$} & EXTR. & \multicolumn{2}{|c|}{63265} & 20543 & 83808 \\
\hline & STRIP & \multicolumn{2}{|c|}{63316} & 13589 & 76905 \\
\hline & BACKW. & \multicolumn{2}{|c|}{62508} & 8627 & 21135 \\
\hline & TOTAL & \multicolumn{2}{|c|}{189089} & 42759 & 231848 \\
\hline \multirow[t]{4}{*}{$5 / 5 / 83$} & EXTR. & 60823 & 3394 & 20997 & 85214 \\
\hline & STRIP & 60357 & 2653 & 13800 & 76810 \\
\hline & BACKW. & 59692 & 2838 & 8942 & 11472 \\
\hline & TOTAL & 180872 & 8885 & 43739 & 233496 \\
\hline \multirow[t]{4}{*}{$9 / 7 / 83$} & EXTR. & 61487 & 3076 & 22412 & 86975 \\
\hline & STRIP & 59529 & 2856 & 15782 & 78166 \\
\hline & BACKW. & 56569 & 2776 & 7674 & 67019 \\
\hline & TOTAL & 177585 & 8708 & 45868 & 232160 \\
\hline \multirow[t]{4}{*}{$12 / 5 / 83$} & EXTR. & 60482 & 2940 & 20539 & 83960 \\
\hline & STRIP & 60128 & 3536 & 13349 & 77013 \\
\hline & BACKW. & 59747 & 2728 & 7338 & 69813 \\
\hline & TOTAL & 180357 & 9204 & 41226 & 230787 \\
\hline
\end{tabular}

"Columa and PUlser contents dumped simul taneously. 
TABLE IX

DUMP INVENTORY RESULTS (GRAMS U)

\begin{tabular}{|c|c|c|c|c|c|}
\hline \multirow[b]{2}{*}{ DATE } & \multirow{2}{*}{ COLUMA } & \multicolumn{4}{|c|}{ COMPONENT } \\
\hline & & COLUMM & PULSER & PLUABIMG & TOTAL \\
\hline \multirow[t]{4}{*}{$2 / 4 / 83^{*}$} & EXTR. & \multicolumn{2}{|c|}{9439} & 2203 & 11642 \\
\hline & STRIP & \multirow{2}{*}{\multicolumn{2}{|c|}{$\begin{array}{l}9217 \\
4370\end{array}$}} & 1526 & 10743 \\
\hline & BACKW. & & & 262 & 5132 \\
\hline & TOTAL & \multicolumn{2}{|c|}{23026} & 4491 & 27517 \\
\hline \multirow[t]{4}{*}{$3 / 4 / 83^{*}$} & EXTR. & \multicolumn{2}{|c|}{5938} & 1563 & 7501 \\
\hline & STRIP & \multicolumn{2}{|c|}{8447} & 1272 & 9719 \\
\hline & BACKW. & \multicolumn{2}{|c|}{3518} & 702 & 4225 \\
\hline & TOTAL & \multicolumn{2}{|c|}{17903} & 3542 & 21445 \\
\hline \multirow[t]{4}{*}{$5 / 5 / 83$} & EXTR. & 3331 & 13 & 1626 & 4970 \\
\hline & STRIP & 9159 & 461 & 1488 & 11108 \\
\hline & BACKW. & 3989 & 285 & 838 & 5112 \\
\hline & TOTAL & 16479 & 759 & 3952 & 21190 \\
\hline \multirow[t]{4}{*}{$9 / 7 / 83$} & EXTR. & 9913 & 355 & 2480 & 12749 \\
\hline & STRIP & 9832 & 512 & 1319 & 11663 \\
\hline & BACKW. & 3782 & 329 & -524 & 4634 \\
\hline & TOTAL & 23527 & 1196 & 4323 & 29046 \\
\hline \multirow[t]{4}{*}{$12 / 5 / 83$} & EXTR. & 8793 & 239 & 2311 & 11343 \\
\hline & STRIP & 8768 & 530 & 1438 & 10736 \\
\hline & BACKW. & 3352 & 281 & 644 & 4277 \\
\hline & TOTAL & 20913 & 1050 & 4393 & 26356 \\
\hline
\end{tabular}

"COLUMn ANd PULSER CONTENTS dumped Simultaneously.

inventory measurements of this strip column. Also shown in Fig. 14 are the upper 300 channels of the same tiree spectra (d, e, and $f$, respectively) with expanded vertical scales. The gains oi the six EUREKA detectors are approximately matched to simplify visual diagnostics.

The net area of the $60-\mathrm{keV}$ peak was determined by straight-1ine background subtraction using two regions of interest, one below and one above the peak region of interest. The net area of the $186-\mathrm{keV}$ peak was determined by subtraction of the toral counts in a single region of interest above the peak, equal in width to that of the peak region of interest.

The presence of three stainless steel pipes or tubes (for aqueous feed, organic sampling, and bubbler manometry) inside the solvent extraction columns, extending from the tops of the columns to below the solution overflow levels, 

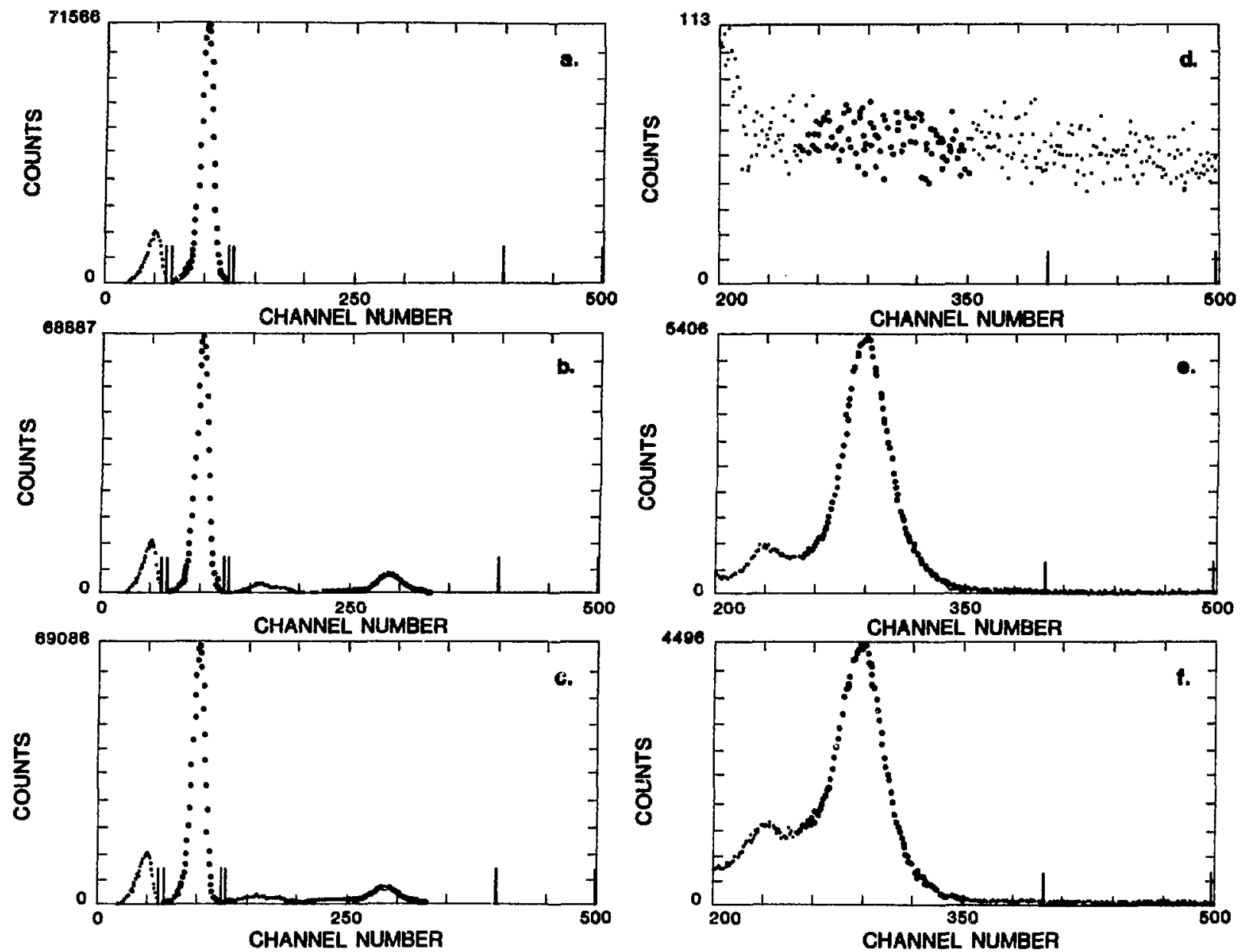

Fig. 14. The detector 5 background ( $a$ and $d$ ), foil (b and $e$ ), and aqueous solution ( $c$ and $f$ ) spectra obtained during the static inventory measurements performed in November 1984. The 60-keV photopeaks are in channels 100 and 290 , respectively. Frames $a, b$, and $c$ show the full spectra, and $d$, $e$, and $f$ show the upper 300 channels. The data points in the peak regions of interest are intensified. The three background regions of interest (below and above the $60-\mathrm{keV}$ peak and above the $186-\mathrm{keV}$ peak) $\mathrm{l}$ ie between the vertical markers.

reduces the 186-keV count rate for detector 6 (positioned to give the concentration of the pure organic phase of each column) by a few per cent from the calculated result. Because geometries of the pipe positions with respect to the detector 6 location vary from column to column and because the effect is concentration dependent, the correction factor for this reduction effect was evaluated empirically. This was done by comparing the measured count rates with those predicted by the calculation for uranium concentrations determined 
by external assay of an organic sample withdrawn from the static organic phase before the static EUREKA measurements commenced. Figure 15 is a plot of the empirically determined correction factors vs uranium concentration for each column. Several data points, all of them larger than average, have been eliminated on statistical grounds from the computed average correction factor for each detector. The anomalously large bias in one of the extraction column data points could have resulted from leaving the manometer air bubbler on during the static EUREKA assay. The lesser anomalies are probably the results of sampling.

Correction factors of $1.066,1.057$, and 1.070 are stored in the EUREKA parameter file for the extraction, strip, and backwash columns, respectively.

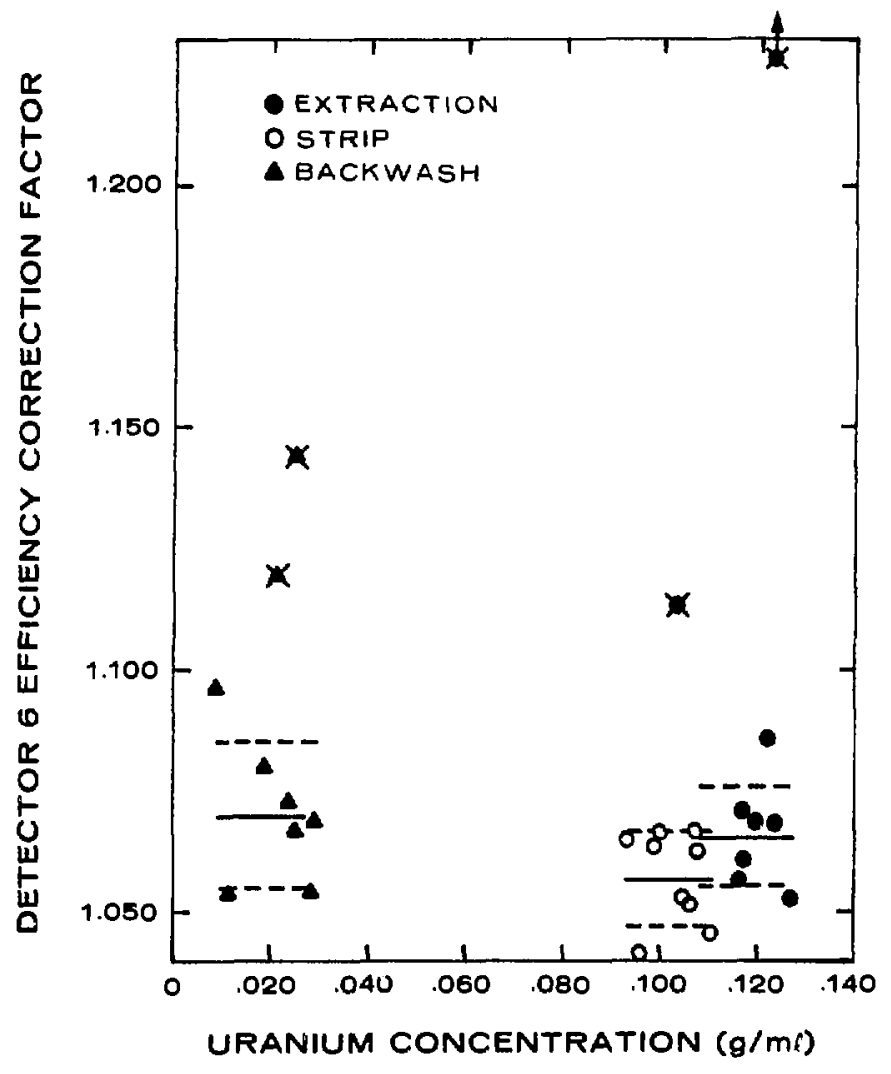

Fig. 15. Correction factor to the detector 6 efficiency factor, deduced empirically for each column, is plotted vs uranium concentration. The mean result and standard deviation are shown for each column by the solid and dashed lines, respectively. Results marked with $X$ were not included in the averaging. 
These are used by the code to multiply the measured count rates for the 186-keV gamma rays in the analysis of the detector 6 result:s to give uranium concentration.

Figures 16-18 are plots of the uranium concentrations vs column height (where zero corresponds to the bottom of the column) measured monthly by EUREKA for the extraction, strip, and backwash columns, respectively. The month designated for each data set is the operation month that precedes the actual EUREKA measurement. The dashed and solid (plus dotted) lines are smiooth curves drawn between the pulsed and static results, respectively. The solid histogram illustrates the method, applied to the static results as shown in Figs. 16-18, used by the EUREKA code to numerically integrate the concentration profiles to give the column inventory. The solid vertical lines at the right of the histo.. gram mark the heights of the aqueous/organic interface and the solution levels

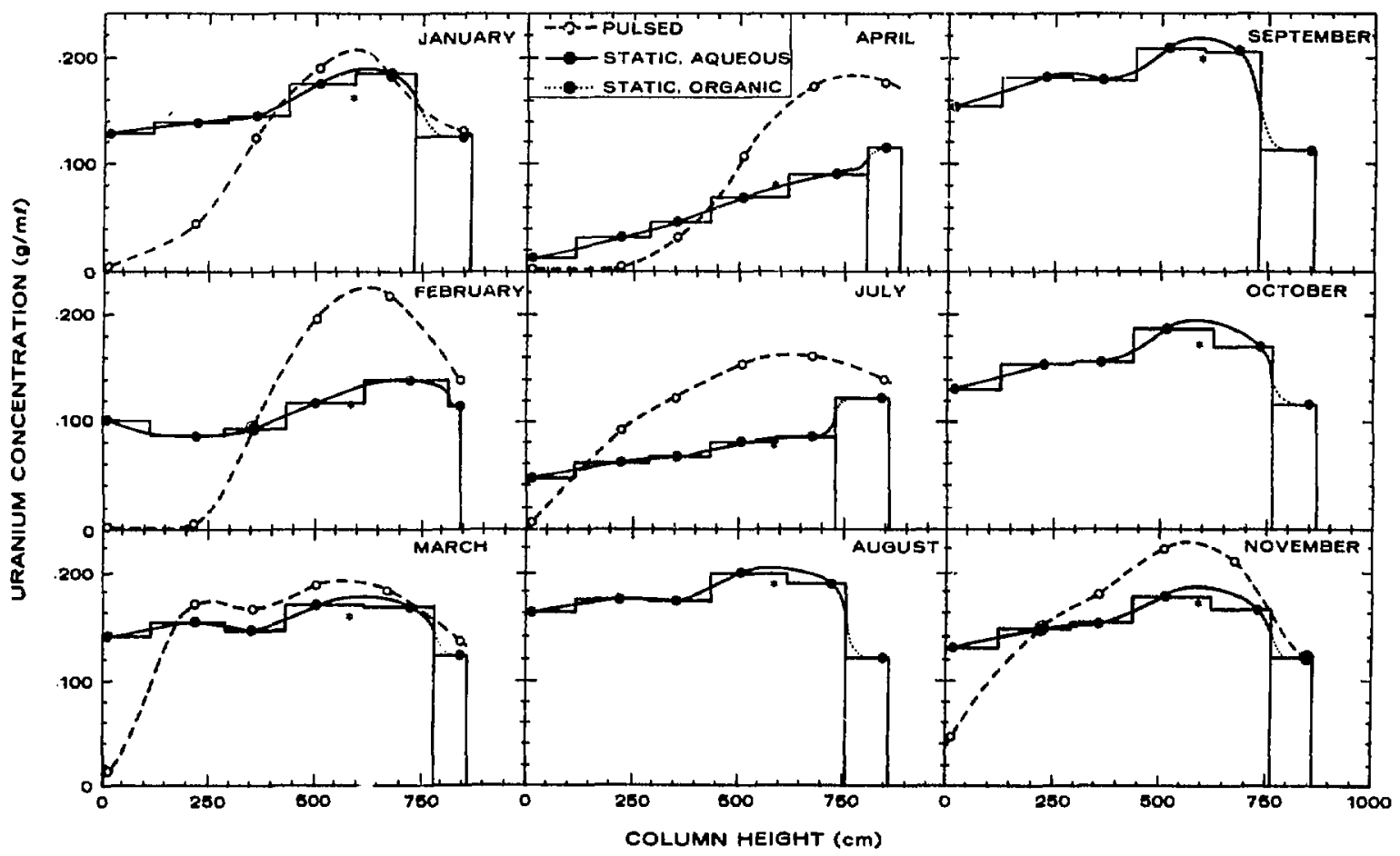

Fig. 16. Uranium concentration plotted vs extraction column height for pulsed and static EUREKA assays at inventory. Smooth curves are drawn tio approximate the pulsed and static profiles. The histogram used by the EUREKA code for inventory determination is also shown. Asterisks are external assay results for samples withdrawn from the static aqueous phase at a fixed position. The plotted results correspond to the 1983 inventory measurements after operation for the indicated months. 


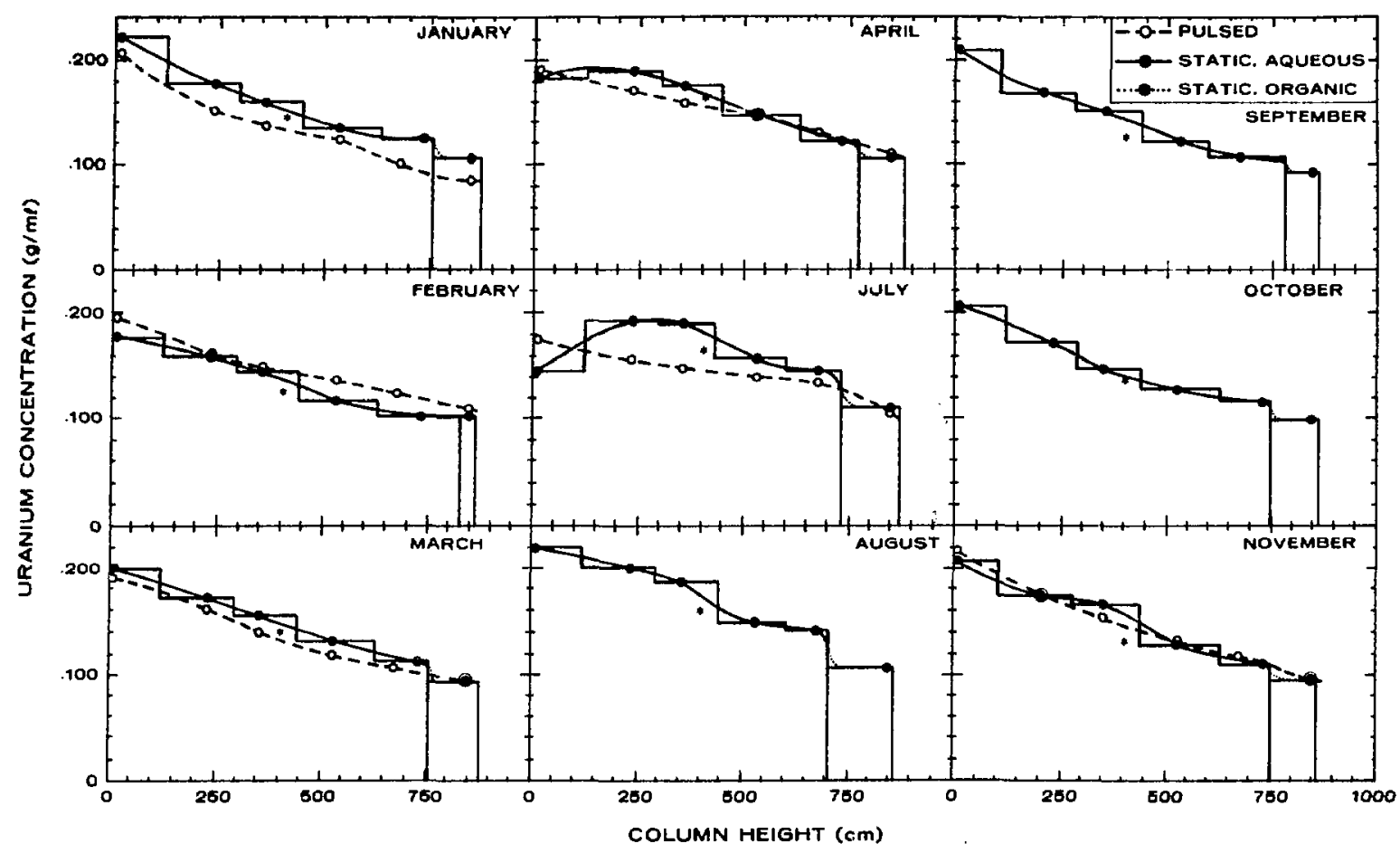

Fig. 17. Uranium concentration plotted vs strip column height for pulsed and static EUREKA assays at inventory. Smooth curves are drawn to approximate the pulsed and static profiles. The histogram used by the EUREKA code for inventory determination is also shown. Asterisks are external assay results for samples withdrawn from the static aqueous phase at a fixed position. The plotted results correspond to the 1983 inventory measurements after operation for the indicated months.

in the static columns. These heights vary from column to column and from month to month. The vertical position of detector 5 (fifth detector from the bottom) differs for the pulsed and static assays for most inventory periods. There are two (alternative) brackets attached to or near each column for mounting detector 5. The lower bracket, at the top of each stainless steel section, is used for all pulsed assays. In the static mode, because of the sharp discontinuity in concentration at the well-defined solution interface, it is desirable to position detector 5 below and as close to this interface as possible. Therefore, a second bracket for detector 5 is mounted for this purpose on the lower portion of the glass section. This alternate mounting position is used when the interface level is relatively high in the glass section. 


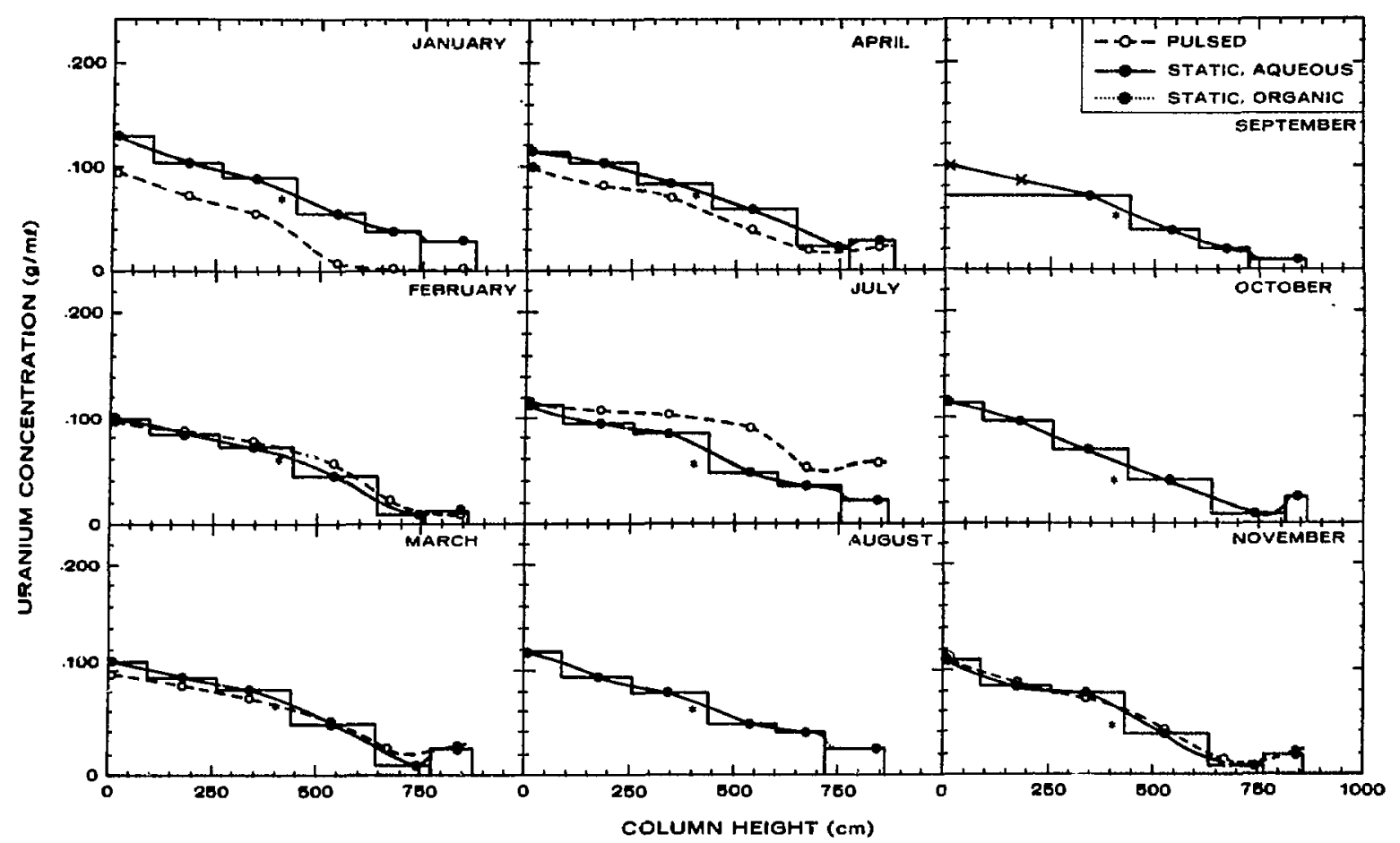

Fig. 18. Uranium concentration plotted vs backwash column height for pulsed and static EUREKA assays at inventory. Smooth curves are drawn to approximate the pulsed and static profiles. The histogram used by the EUREKA code for inventory determination is also shown. Asterisks are external assay results for samples withdrawn from the static aqueous phase at a fixed position. The plotted results correspond to the 1983 inventory measurements after operation for the indicated months.

Large differences in the column concentration profiles for the pulsed and static measurements, particularly for the extraction column, illustrate the need for using pulsed assays to obtain concentration information that relates to the piumbing contents. The detector 1 (bottom) and detector 6 (top) assay results are used for these purposes. Detector 1 is positioned below the organic feed line in the inactive region of each column. Therefore, it assays a pure aqueous solution during puised operation, and the concentration is representative of the aqueous solution drain components of the external plumbing for a given column. Detector 6 is mounted above the aqueous feed line in the inactive region of each column. Therefore, it assays a pure organic solution during pulsed operation, and the concentration is representative of the organic solution overflow components of the external plumbing for a given column. 
No user input is required for the pulsed EUREKA assays, other than for column identification. For the static EUREKA assays, input of the heights, A and $B$, of the total column solution and phase interface, respectively, is required for each column. These levels are read and recorded routinely by the process operator at each inventory period after system shutdown and pulsing. The EUREKA code applies these quantities to the calculation of organic and aqueous solution volumes. The A level varies from month to month because samples are routinely pulled from the aqueous and organic phases following pulsing for phase disengagement. Because the EUREKA inventories were compared with the dump inventories, the $A$ level after sampling was the value required by the code to compute the comparable solution volume. The B level also changes after sampling, but bigger factors in this change from month to month are the column operation parameters. Furthermore, should any shutdown (followed by pulsing) result in a $B$ (interface) level below the glass sections, acid solution (free of uranium) is added to the column to raise the $B$ level to make it visible within the glass section. In all cases, it was essential for valid comparisons that the reading of $A$ and $B$, as well as the static EUREKA assays, follow the ac:d addition and sample withdrawal processes.

Because the process operator routinely samples (for external assay) the aqueous phase after shutdown and pulsing for phase disengagement, the EUREKA code is designed to optionally use this assay information (uranium concentration and specific gravity) to compute the acid molarity of the aqueous solutions in the column. Preliminary sample measurements showed acid molarity variations of as much as $\pm 2 M$ about a mean of $3.5 \underline{M}$ in the extraction column. In this range $(1.5 \mathrm{M}$ to $5.5 \mathrm{M})$, the change in $186-\mathrm{keV}$ count rate for $100 \mathrm{~g} \mathrm{U} / \mathrm{l}$ is $22 \%$. This propagates to a $4 \%$ effect in the uranium concentration assay. A11 EUREKA inventory results determined during the operational evaluation were obtained by using the sample assay result in correcting for acid molarity in the extraction column. The measured variation in acid molarity at the extraction column sampling point was $\pm 0.8 \underline{M}( \pm 1 \sigma)$, about a mean value of $3.7 M$. This is less than one-half of the variation originally predicted by the preliminary extraction column sample measurements. In the absence of this input to the EUREKA code, the default values used by the code would give rise to maximum errors that are documented in Table II. 
The sample assay results for uranium concentration are plotted at fixed heights as asterisks in Figs. 16-18. These heights correspond to the vertical locations of the aqueous sample withdrawal spigots on the columns.

Tables $X$ and $X I$ give the dates and results of EUREKA measurements of solution volumes and uranium masses, respectively, for those inventories that were foll lowed by direct measurements. Tabies $X I I(A)$ and $X I I(B)$ give the ratios of the EUREKA volumes to the reference volumes of the analogous dump components. (These are the ratios of the results in Table $x$ to those in Table VIII.) The means and standard deviations in these quantities are given in Tables XIII(A) and XIII(B). Tables XIV(A) and XIV(B) give the ratios of the EUREKA uranium masses to the reference uranium masses of the analogous dump components. (These are the ratios of the results of Table XI to those of Table IX.) The means and standard deviations in these quantities are given in Tables $X V(A)$ and $X V(B)$. The $(A)$ and $(B)$ distinctions in Tables $X I I-X V$ permit evaluation of the EUREKA assumptions concerning the pulser line for the last three inventory comparisons in which the colurin and pulser solution components were dumped separately.

\section{RESULTS OF EVAPORATOR MONITOR EVALUATION}

Each 30-s spectrum of evaporator solutions is acquired in 1024 channels of multichannel analyzer memory. The spectra (solution, as well as background and foill obtained by the evaporator monitor are qualitatively and quantitatively like those in Fig. 14. The regions of interest and the methods for obtaining peak areas are the same as those used by the EUREKA instrument.

The uranium concentration monitor for the $Y-12$ secondary intermediate evaporator has functioned since installation as a device for continuous readout of uranium concentration of the evaporator solutions. The monitor is used by the operator responsible for manual control of the evaporator. During several periods of monitor operation, samples were withdrawn from the evaporator return 10op. The external analysis of the samples by nondestructive high-resolution gamma-ray assay ${ }^{2,7}$ gave uranium concentrations plotted in Figs. 19-21 (as solid points) along with the monitor results (circles) at the approximately 1 -min assay cycle intervals. The percentage deviation between the monitor 
TABLE $X$

EUREKA VOLUMES (MILLILITERS)

\begin{tabular}{|c|c|c|c|c|c|}
\hline \multirow[b]{2}{*}{ DAIE } & \multirow{2}{*}{$\frac{\text { COLUKK }}{10}$} & \multicolumn{4}{|c|}{ COMPONENT } \\
\hline & & COLUAM & PULSER * & PLIMRIHG: & IOJAL \\
\hline \multirow[t]{4}{*}{$2 / 4 / 83$} & EXTR. & 59633 & 2555 & 20423 & 82611 \\
\hline & STRIP & 59924 & 2555 & 15772 & 78251 \\
\hline & BACKW. & 60360 & 2555 & 8691 & 71606 \\
\hline & TOTAL & 179917 & 7665 & 44886 & 232468 \\
\hline \multirow[t]{4}{*}{$3 / 4 / 83$} & EXTR. & 58176 & 2555 & 20423 & 81154 \\
\hline & STRIP & 59391 & 2555 & 15772 & 77718 \\
\hline & BACKW. & -59294 & 2555 & 8691 & -70540 \\
\hline & TOTAL & 176861 & 7665 & 44886 & 229412 \\
\hline \multirow[t]{4}{*}{$5 / 5 / 83$} & -EXIR. & 60845 & 2555 & 20423 & 83823 \\
\hline & SIRIP & 60651 & 2555 & 15772 & 78978 \\
\hline & BACKW. & 60599 & 2555 & 8691 & 71945 \\
\hline & TOTAL & 182195 & 7665 & 44886 & 234746 \\
\hline \multirow[t]{4}{*}{$9 / 7 / 83$} & EXTR. & 59100 & 2555 & 20423 & 82078 \\
\hline & STRIP & 59197 & 2555 & 15772 & 77524 \\
\hline & BACKW. & 59343 & 2555 & 8691 & 20589 \\
\hline & TOTAL & 177640 & 7665 & 44886 & 230191 \\
\hline \multirow[t]{4}{*}{$12 / 5 / 83$} & EXTR. & 59294 & 2555 & 20423 & 82272 \\
\hline & STRIP & 59294 & 2555 & 15772 & 77621 \\
\hline & BACKW. & 59343 & 2555 & 8691 & 70589 \\
\hline & TOTAL & 177931 & 7665 & 44886 & 230482 \\
\hline
\end{tabular}

*BASED OH CALCULATED COLUMH VOLUMES (USING DATA FROM ENGINEERIHG DRAWINGS) AKD ON OPERATOR INPUT OF HEIGHT OF SOLUTION AFTER SAMPLE WI IHDRAWAL.

* Based on calculated volumes (USing measurej PIPE lengths and diamEIERS).
TABLE XI

EUREKA INVENTORY RESULTS (GRAMS U)

\begin{tabular}{|c|c|c|c|c|c|}
\hline \multirow[b]{2}{*}{ DATE } & \multirow{2}{*}{$\begin{array}{c}\text { COLUMA } \\
\text { ID }\end{array}$} & \multicolumn{4}{|c|}{ COMPONENT } \\
\hline & & Columer & PULSER & PLUMBING & IOIAL \\
\hline \multirow[t]{4}{*}{$2 / 4 / 83$} & EXTR. & 9017 & 10 & 1836 & 10863 \\
\hline & STRIP & 9156 & 526 & 1648 & 11330 \\
\hline & BACKW. & -4339 & 242 & 569 & 5150 \\
\hline & TOTAL & 22512 & 778 & 4053 & 27343 \\
\hline \multirow[t]{4}{*}{$3 / 4 / 83$} & EXTR. & 6497 & 4 & 1947 & 8448 \\
\hline & STRIP & 7955 & 501 & 1946 & 10402 \\
\hline & BACKW. & 3148 & 247 & 600 & 3995 \\
\hline & TOTAL & 17600 & 752 & 4493 & 22845 \\
\hline \multirow[t]{4}{*}{$5 / 5 / 83$} & EXTR. & 3847 & 10 & 2486 & 6343 \\
\hline & STRIP & 9357 & 489 & 1924 & 11770 \\
\hline & BACKW. & 4039 & 251 & 646 & -4936 \\
\hline & TOTAL & 17243 & 750 & 5056 & 23049 \\
\hline \multirow[t]{4}{*}{$9 / 7 / 83$} & EXTR. & 10074 & 16 & 1986 & $12076]$ \\
\hline & STRIP & 9756 & 445 & 1830 & 12031 \\
\hline & BACKW. & 3803 & 294 & 842 & 4939 \\
\hline & TOTAL & 23633 & 755 & 4658] & 29046 \\
\hline \multirow[t]{4}{*}{$12 / 5 / 83$} & EXTR. & 8920 & 111 & 1977 & 11008 \\
\hline & SIRIP & 8659 & 554 & 1810 & 11023 \\
\hline & BACKW. & 3295 & 282 & 712 & 4294 \\
\hline & TOTAL & 20874 & 947 & 4504 & 26325 \\
\hline
\end{tabular}

"EUREKA ASSAYS DURING PULSED OPERATION WERE NOT PERFORMED IN SEPIEMBER. THEREFORE, EUREKA CODE AUTOMATICALLY USES THE CONCENTRATIONS THIS CASE, 8/4/83) TO OBTAIN PLUMBING INVENTORIES. 
TABLE XII (A)

(EUREKA VOLUHE)/(DUMP VOLUME)

\begin{tabular}{|c|c|c|c|c|}
\hline \multirow[b]{2}{*}{ DAIE } & \multirow{2}{*}{$\begin{array}{c}\text { COLUAN } \\
\text { ID }\end{array}$} & \multicolumn{3}{|c|}{ COMPONENI } \\
\hline & & SOLUMN + PULSER & PLUMBING & TOTAL \\
\hline \multirow[t]{4}{*}{$2 / 4 / 83^{*}$} & EXTR. & 0.962 & 1.018 & 0.975 \\
\hline & STRIP & 0.982 & 1.149 & 1.011 \\
\hline & BACKH. & 0.992 & 1.035 & 0.997 \\
\hline & TOTAL & 0.978 & 1.064 & 0.994 \\
\hline \multirow[t]{4}{*}{$3 / 4 / 83^{*}$} & EXTR. & 0.960 & 0.994 & 0.968 \\
\hline & STRIP & 0.978 & 1.161 & 1.011 \\
\hline & BACKW. & 0.989 & 1.002 & Q.992 \\
\hline & TOTAL & 0.976 & 1.050 & 0.989 \\
\hline \multirow[t]{4}{*}{$5 / 5 / 83^{* *}$} & EXIR. & 0.987 & 0.973 & 0.984 \\
\hline & STRIP & 1.003 & 1.143 & 1.028 \\
\hline & BACKW. & 1.012 & 0.972 & 1.007 \\
\hline & TOTAL & 1.001 & 1.026 & 1.005 \\
\hline \multirow[t]{4}{*}{$9 / 7 / 83^{* *}$} & EXTR. & 0.955 & 0.911 & 0.944 \\
\hline & STRIP & 0.990 & 0.999 & 0.992 \\
\hline & BACKH. & 1.043 & 1.133 & 1.053 \\
\hline & TOTAL & 0.995 & 0.979 & 0.992 \\
\hline \multirow[t]{4}{*}{$12 / 5 / 83^{*} *$} & EXTR. & 0.975 & 0.994 & 0.980 \\
\hline & SIRIP & 0.971 & 1.182 & 1.008 \\
\hline & BACKH. & e.991 & 1.184 & 1.011 \\
\hline & TOTAL & 0.979 & 1.089 & 0.999 \\
\hline
\end{tabular}

TABLE XII(B)

(EUREKA VOLUME)/(DUMP VOLUME) *

\begin{tabular}{|c|c|c|c|}
\hline \multirow[b]{2}{*}{ DAIE } & \multirow{2}{*}{$\begin{array}{c}\text { COLUHA } \\
\text { ID }\end{array}$} & \multicolumn{2}{|r|}{ COMPOMENI } \\
\hline & & COLUHA & PULSER \\
\hline \multirow[t]{4}{*}{$5 / 5 / 83$} & EXTR. & 1.000 & 0.753 \\
\hline & STRIP & 1.005 & 0.963 \\
\hline & BACKW. & 1.012 & 0.900 \\
\hline & TOTAL & 1.007 & 0.863 \\
\hline \multirow[t]{4}{*}{$9 / 7 / 83$} & EXTR. & 0.961 & 0.831 \\
\hline & SIRIP & 0.994 & 0.895 \\
\hline & BACKW. & 1.049 & 0.920 \\
\hline & TOTAL & 1.000 & 0.880 \\
\hline \multirow[t]{4}{*}{$12 / 5 / 83$} & EXTR. & 0.980 & 0.865 \\
\hline & STRIP & 0.986 & $0 .-23$ \\
\hline & BACKW. & 0.993 & 0.937 \\
\hline & TOTAL & 0.987 & 0.833 \\
\hline $\begin{array}{l}\text { PANALYSIS } \\
\text { PERIODS } \\
\text { RATELY FF }\end{array}$ & \multicolumn{3}{|c|}{$\begin{array}{l}\text { RESULTS FROM LAST THREE INVEHTORY } \\
\text { PULSER COMPOHENTS HERE DUMPED SEPA- } \\
\text { COLUMH COMPONENTS. }\end{array}$} \\
\hline
\end{tabular}

* Columa amd pulser contents dumped simul tameously.

* SEPARATEd COLUMA AND PULSER COMPOHENTS COMBIMED FOR thIS aHalysis. 
TABLE XIII(A)

AVERAGE VALUES $(\mathbf{X 1 0 )}$ * OF (EUREKA VOLUME)/(DUMP VOLUME)

\begin{tabular}{lccc}
$\begin{array}{c}\text { COLUMM ID } \\
\text { (WO. AVERAGED) }\end{array}$ & COLUMH + PULSER & PLUMBING & TOTAL \\
\hline EXTR. (5) & $0.968(1.3 \times)$ & $0.978(4.2 x)$ & $0.970(1.6 x)$ \\
STRIP (5) & $0.985(1.2 x)$ & $1.127(6.4 x)$ & $1.010(1.3 x)$ \\
BACKH. (5) & $1.005(2.3 x)$ & $1.066(8.3 x)$ & $1.012(2.4 x)$ \\
ALL (15) & $0.986(2.2 x)$ & $1.057(8.6 x)$ & $0.997(2.6 x)$ \\
TOTALS (5) & $0.986(1.1 x)$ & $1.042(4.0 x)$ & $0.996(0.6 x)$
\end{tabular}

"Sample relative standard deviation.

- "SEPARATEd COLUMh AND PULSER COMPONENTS COMBIHEd for this analysis.
TABLE XIII (B)

AVERAGE VALUES $\left(x_{1} \sigma\right)$ * OF (EUREKA VOLUME)/(DUMP VOLUME)**

$\begin{array}{lll}\begin{array}{c}\text { COLUMN ID } \\ \text { (NO. AVERAGED) }\end{array} & \text { COLUHN } & \text { PULSER } \\ \text { EXTR. (3) } & 0.980(2.0 x) & 0.818(7.2 x) \\ \text { SIRIP (3) } & 0.995(1.0 x) & 0.860(14.4 x) \\ \text { BACKW. (3) } & 1.020(2.7 x) & 0.919(19.6 x) \\ \text { ALL (9) } & 0.998(2.5 x) & 0.866(9.5 x) \\ \text { TOTALS (3) } & 0.998(1.0 x) & 0.859(2.8 x)\end{array}$

- SAMPLE RELATIVE STANDARD DEVIATION.

- "LAST THREE INVENTORY PERIODS WHEN PULSER COM-

PONENTS WERE DUMPED SEPARATELY FROM COLUMN COH-

PONENTS. 
TABLE XIV(A)

(EUREKA INVERTORY)/(DUMP INVENTORY)

\begin{tabular}{|c|c|c|c|c|}
\hline \multirow[b]{2}{*}{ DATE } & \multirow{2}{*}{ COLUMM } & \multicolumn{3}{|c|}{ COMPONENI } \\
\hline & & COLUMH \pm PULSER & PLUMBING & TOLAL \\
\hline \multirow[t]{4}{*}{$2 / 4 / 83^{*}$} & EXTR. & 0.956 & 0.833 & 0.933 \\
\hline & STRIP & 1.050 & 1.080 & 1.055 \\
\hline & BACKH. & 1.048 & 0.747 & 1.004 \\
\hline & TOTAL & 1.011 & 0.902 & 0.994 \\
\hline \multirow[t]{4}{*}{$3 / 4 / 83^{*}$} & EXTR. & 1.095 & 1.246 & 1.126 \\
\hline & STRIP & 1.001 & 1.530 & 1.070 \\
\hline & BACKW. & 2.965 & 0.849 & 0.946 \\
\hline & TOTAL & 1.025 & 1.268 & 1.065 \\
\hline \multirow[t]{4}{*}{$5 / 5 / 83^{* *}$} & EXTR. & 1.154 & 1.529 & 1.276 \\
\hline & STRIP & 1.023 & 1.293 & 1.060 \\
\hline & BACKW. & 1.004 & 0.771 & 0.956 \\
\hline & TOTAL & 1.044 & 1.279 & 1.088 \\
\hline \multirow[t]{4}{*}{$9 / 7 / 83^{* \prime}$} & EXTR. & 0.983 & {$[0.801]$} & 0.947 \\
\hline & STRIP & 0.986 & 1.387 & 1.032 \\
\hline & BACKW. & 0.997 & 1.602 & 1.066 \\
\hline & TOTAL & 0.988 & 1.077 & 1.000 \\
\hline \multirow[t]{4}{*}{$12 / 5 / 83^{* * *}$} & EXTR. & 1.000 & 0.855 & 0.970 \\
\hline & STRIP & 0.991 & 1.259 & 1.027 \\
\hline & BACKW. & 0.985 & 1.113 & 1.004 \\
\hline & TOTAL & 0.994 & 1.025 & 0.999 \\
\hline
\end{tabular}

"COLUNH AND PULSER CONTENTS DUMPED SIMUL TANEOUSLY.

* "SEPARATEd COLUma and pUlser components COMBIHEd for this amalysis.

BER THEREFORE DUREKA PULSED OPERAT ION WERE NOT PERFORMED IN SEPTEM

BER.

CASE. 8/4/831 TO OBTAIN PLUMBIMG INUEMTORIES.

U
TABLE XIV(B)

(EUREKA INVENTORY)/(DUMP INVENTORY)*

$\left.\begin{array}{llll} & \text { COLUAH } & \multicolumn{2}{c}{\text { COMPONENI }} \\ \text { DAIE } & \text { COLUMK } & \text { PULSER } \\ 5 / 5 / 83 & \text { EXTR. } & 1.155 & 0.769 \\ & \text { STRIP } & 1.022 & 1.061 \\ & \text { BACKH. } & 1.013 & 0.881 \\ & \text { TOTAL } & 1.046 & 0.988 \\ 9 / 7 / 83 & \text { EXIR. } & 1.016 & 0.045 \\ & \text { STRIP } & 0.992 & 0.869 \\ & \text { BACKH. } & 1.006 & 0.894 \\ & \text { TOTAL } & 1.005 & 0.631\end{array}\right]^{n *}$

12/5/83 EXIR $\quad 1.014 \quad 0.464$

STRIP $\quad 0.988 \quad 1.045$

BACKW. $\quad 0.983 \quad 1.004$

TOTAL $\quad 0.997 \quad 0.902$

- ANaLysis of resUl tS from last three INVENTORY PERIODS WHEN PULSER COMPONENTS WERE DUHPED SEPARAIELY FROM COLUMA COMPONENTS. RATELY FROM COLUMH COMPONENTS.

PERFORHED IN SEPIEHER PULSE OPERATION WERE HOT PERFORMED IN SEP IEMEER. THEREFORE, EUREKA CODE FROM PULSED ASSAYS PERFORHED DURING THE PREVIOUS MONTH (III THIS CASE, 8/4/83) TO OBTAII PLUMBING

INVENTORIES. 
TABLE XV(A)

AVERAGE VALUES $(\times 10)$ *F (EUREKA INVENTORY)/(DUMP INVENTORY)

\begin{tabular}{|c|c|c|c|}
\hline $\begin{array}{l}\text { COLUMA ID } \\
\text { (Ne. AVERAGED) }\end{array}$ & COLUMA + PULSER $=$ & PLUMBing & IOTAL \\
\hline EXTR. (5) & $1.038(8.1 x)$ & $1.053(30.6 x)$ & $1.050(14.1 x)$ \\
\hline STRIP (5) & $1.010(2.6 x)$ & $1.310(12.7 x)$ & $1.049(1.8 x)$ \\
\hline BACKW. (5) & $1.000(3.1 x)$ & $1.017(35.48)$ & $0.997(4.6 \%)$ \\
\hline ALL (15) & $1.016(5.1 x)$ & $1.127(27.1 x)$ & $1.032(8.4 \%)$ \\
\hline$(14)^{* * *}$ & $1.006(3.7 x)$ & $1.098(26.8 x)$ & $1.014(5.6 \%)$ \\
\hline TOTALS (5) & $1.012(2.3 x)$ & $1.110(14.6 x)$ & $1.029(4.2 x)$ \\
\hline
\end{tabular}

- Sample Relative standard deviation.

* "Separated coluhn and pulsen components combined for this analysis.

* "OMIT 5/5/83 EXTRACTION C.OLUMN RESULT ON SIATISTICAL GROUNDS.

TABLE XV(B)

AVERAGE VALUES $(\times 10)$ * OF (EUREKA INVENTORY)/(DUMP INVENTORY) * *

\begin{tabular}{|c|c|c|}
\hline $\begin{array}{l}\text { COLUMN ID } \\
\text { (NO. AYERAGED) }\end{array}$ & CoLUMN & PULSER \\
\hline EXTR. (3) & $1.061(7.6 x)$ & $0.426(85.2 x)$ \\
\hline STRIP (3) & $1.001 \quad(1.9 x)$ & $0.992(10.8 x)$ \\
\hline BACKW. (3) & $1.001(1.6 \%)$ & $0.926(7.3 x)$ \\
\hline (9) & $1.021(5.1 x)$ & $0.781(42.3 x)$ \\
\hline 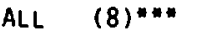 & $1.004(1.5 \%$ & $0.783(45.1 x)$ \\
\hline TOTALS(3) & $1.016(2.6 x)$ & $0.840(22.1 x)$ \\
\hline
\end{tabular}

" SaMple relative standard deyiation.

* "LAST THREE INVENTORY PERIODS WHEN PULSER COMPONENTS WERE DUMPED SEPARATELY FROM COLUMN COMPONENTS.

* *OMIT 5/5/83 EXTRACTION COLUAN RESULT ON STATISTICAL GROUNDS. 


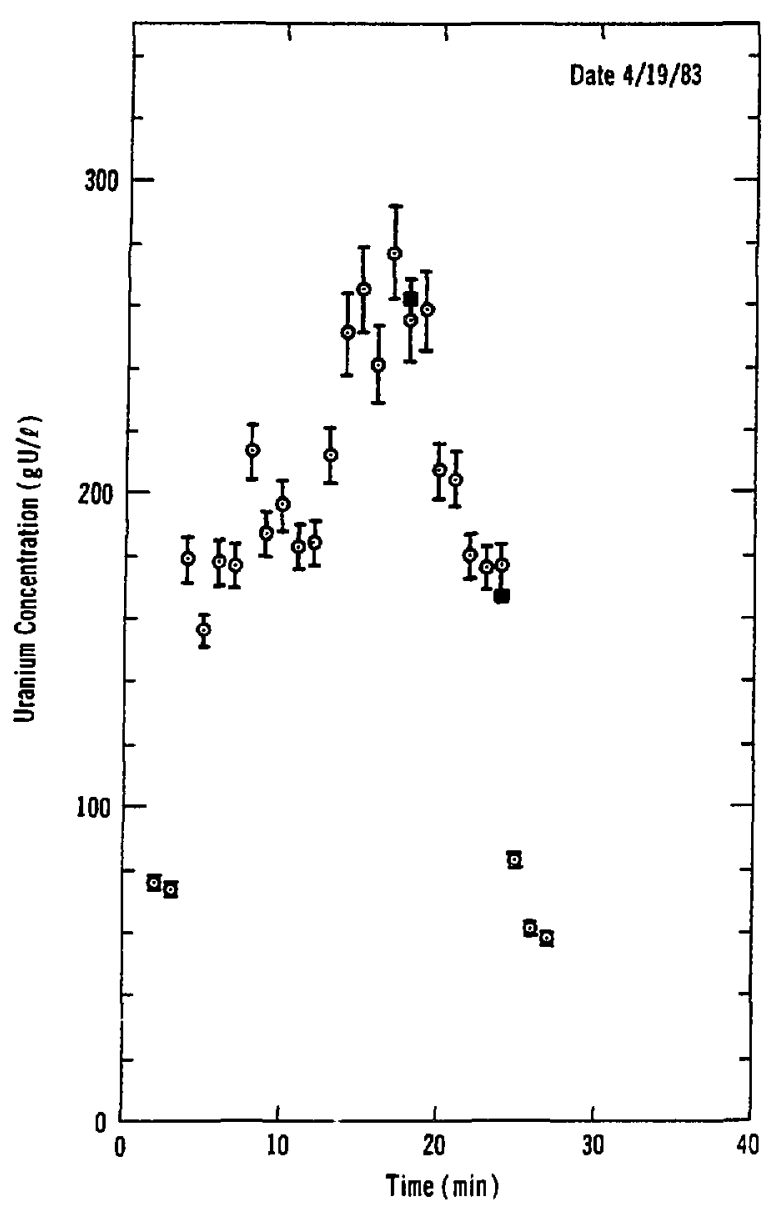

Fig. 19. Evaporator monitor results for uranium concentration plotted vs time. The results of the external analysis of two samples withdrawn from the evaporator return loop are also plotted at the time of sample withdrawal (solid points). The error bars are calculated from the counting statistics $(1 \sigma)$ of the monitor assays.

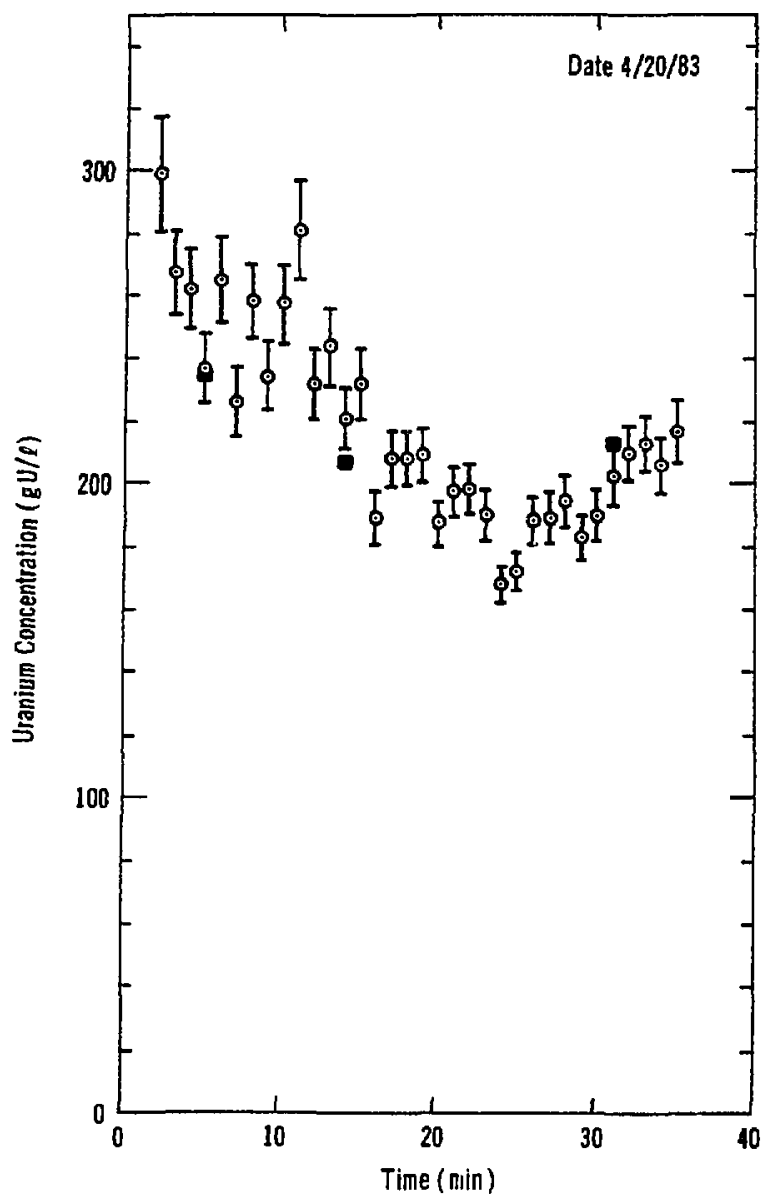

Fig. 20. Evaporator monitor results for uranium concentration plotted vs time. The results of the external analysis of three samples withdrawn from the evaporator return loop are also plotted at the time of sample withdrawal (solid points). The error bars are calculated from the counting statistics $(1 \sigma)$ of the monitor assays.

result (closest in time to that of sample withdrawal) and the sample assay is plotted vs sample assay result in Fig. 22. These data indicate an average bias in the monitor of $21 \%$, with a relative standard deviation of $5 \%$ in the data and $2 \%$ in the mean. The $5 \%$ sample standard deviation agrees with the magnitude of the predicted random error $(1 \sigma)$ in an individual monitor assay (error bars in Figs. 19-21) based on counting statistics. 
ㅁ

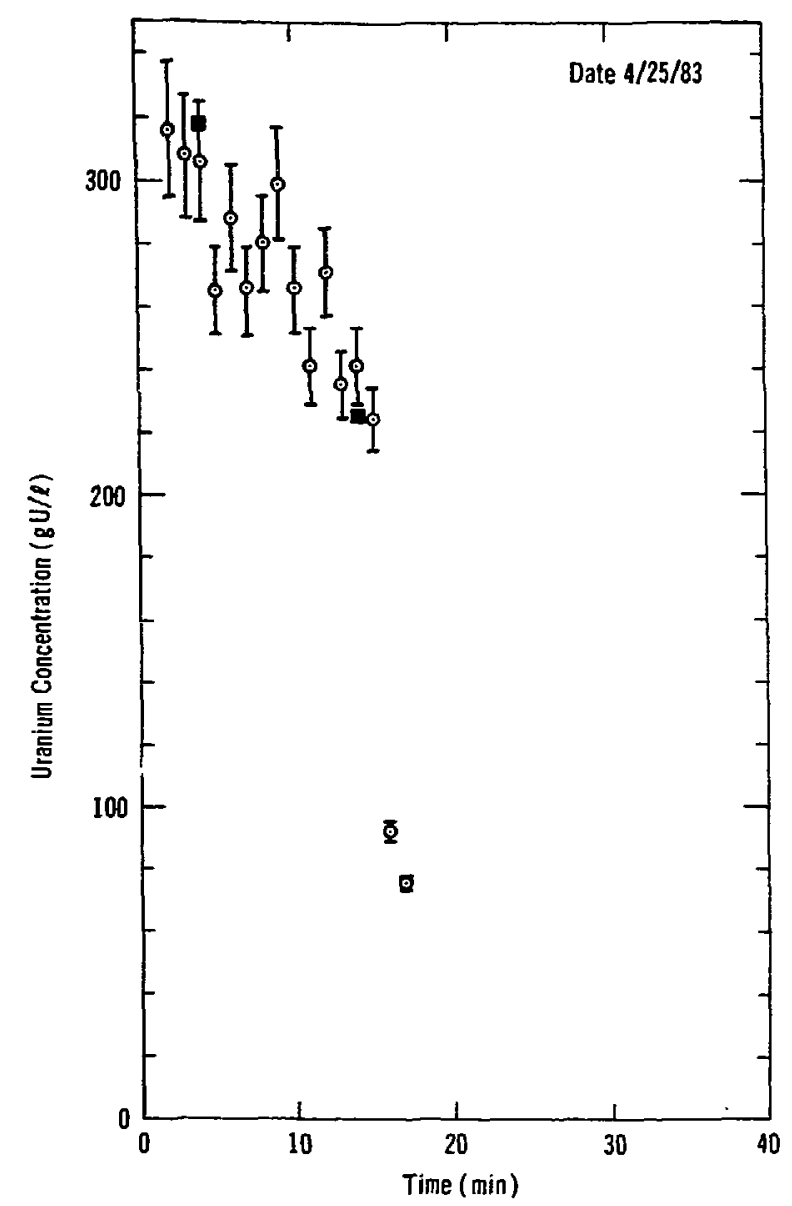

Fig. 21. Evaporator monitor results for uranium concentration plotted vs time. The results of the external analysis of two samples withdrawn from the evaporator return loop are also plotted at the time of sample withdrawal (solid points). The error bars are calculated from the counting statistics (10) of the monitor assays.

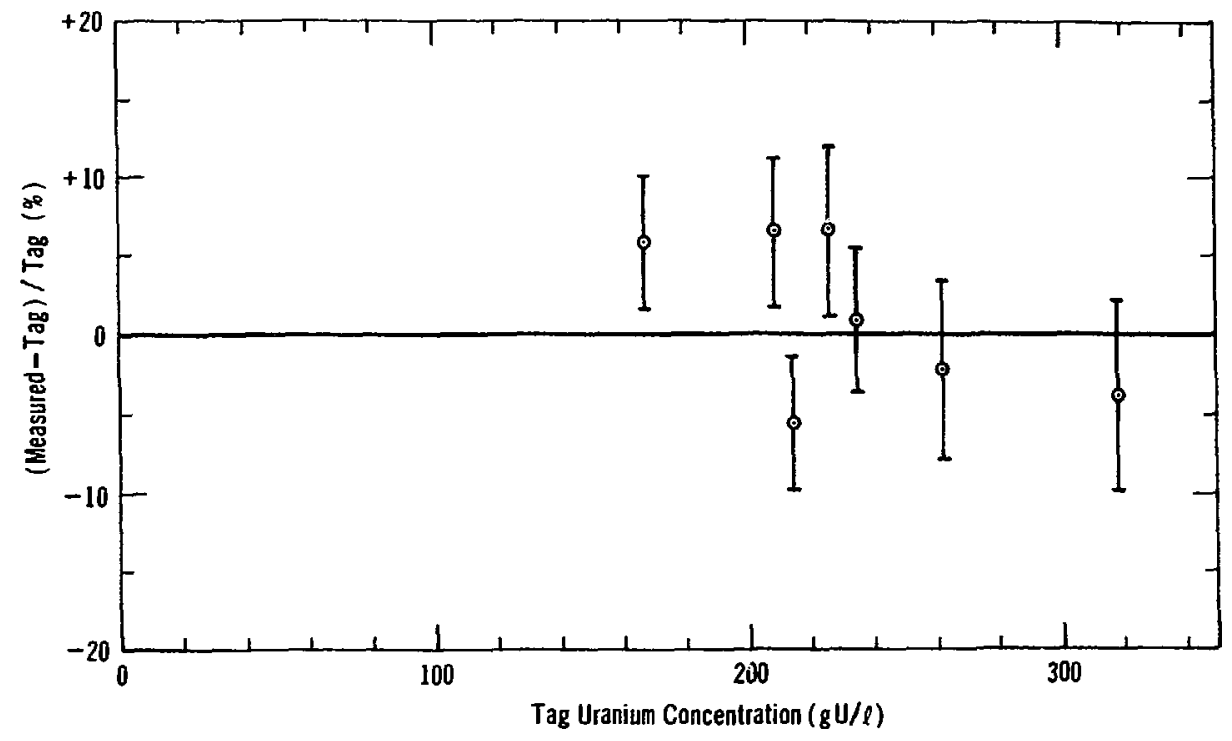

Fig. 22. Percentage deviation between evaporator monitor result (measurement) and external sample assay result (tag) plotted vs external sample assay result for the seven withdrawn samples indicated in Figs. 19-21. The error bars are calculated from the counting statistics (10) of the single monitor assay concurrent with the sample withdrawal. 


\section{v. EVALUATION OF EUREKA RESULTS}

\section{A. Column and Pulser Inventories}

The results of the rigorous measurement control program exercised during the first year of operational evaluation of EUREKA have generated cunfidence in the ability to establish and maintain an accurate calibration for the concentration assay. Comparison of the EUREKA inventory results for the column plus pulser components with the 15 direct measurements of these components indicates an average deviation, column by column, of $1.6 \%$ in the EUREKA inventories [Table XV(A)]. The standard deviation in the relative results, column by column, is $5.1 \%$.

The largest deviation in the EUREKA column-plus-pulser inventory is observed for the extraction column--in particular, for the May 1983 measurements for which the EUREKA result was $15 \%$ larger than the direct result [Table XIV(A) or XiV(B)]. Elimination of the May 1983 measuremant results in an average deviation, column by column, of $0.6 \%$ and a standard deviation, column by column, of 3.7\%. Elimination of the May 1983 extraction column result is justifiad statistically because the deviation of this result from the direct measurements exceeds by $4 \sigma$ the average deviation computed for the other 14 results. An unscheduled addition of solution to the extraction column laqueous raffinate, for example, for storage convenience) between the time of the static EUREKA measurements and the dumps could be the cause of the anomalously large deviation observed. However, no procedural changes have been documented that might account for an invalid result in the May 1983 extraction column measurements.

The measured standard deviation of $3.7 \%$ is substantially larger than the $1 \%$ random uncertainty prediction for the EUREKA inventory of a given column. The prediction is based on the known, quantifiable effects of counting siatistics, variable acid molarity, and random positioning of detectors with respect to the sieve plates (Sec. II.C). However, $3.7 \%$ is the standard deviation in the EUREKA result relative to the dump result; therefore, it includes random effects associated with dumping the columns, sampling the dumped components, and performing external assays of the components. Similarly, the $0.6 \%$ apparent bias includes any systematic effects in these processes of direct verification. The possible random and systematic effects from the direct verification procedures can arise from, among other things, recording of liquid levels before 
(rather than after) sampling lines are sparged and samples are withdrawn, roglecting to isolate (and xeep isolated from the columns) the external plumbing lines after shutdown of the columns, and permitting solution addition to or removal from the columns between the EUREKA assays and the dumps. (The addition of acid or deionized water to the columns to raise the interface level in the column is a routine practice during inventory.)

The average deviation in the total (extraction, strip, and backwash combined) results for columns plus pulsers over the five inventory comparisons is $1.2 \%$. The standard deviation in the relative totals for columns plus pulsers is $2.3 \%$ [Table XV(A)].

An evaluation of the EUREKA inventories for the separated column and pulser components [Table XV(B)] reveals large deviations in the EUREKA results for the pulser lines, particularly for the extraction column. The pulser line inventories, as well as the inventories for all other external plumbing lines in the solvent extraction system, were deduced by EUREKA from concentration and volume measurements performed on the columns. In particular, the volume of solution in each pulser line was computed from the known pipe diameter of the pulser line that extends from the base to above the top of each solvent extraction column and the measured height of solution in the solvent extraction column. The concentration of uranium in the pulser line solution was assumed to be the detector 1 pulsed concentration assay.

The assumption that the liquid level heights are the same in the pulser line and the column is valid only if the average solution densities are the same. Because the solution in the column consists of two distinct phases and because there are steep gradients in uranium concentration from the top of the column to the bottom (in the extraction column in particular), the average solution density in the pulser line can differ significantly from that in the column. The extraction column is the most outstanding example. Furthermore, the detector 1 pulsed concentration assays are representative of uranium concentration in the pulser line during pulsed operation. However, after shutdown and pulsing of the static system for phase separation, equilibration of uranium concentration within the aqueous phase changes the uranium concentration at the detector 1 location (and also in the pulser line), particularly for the extraction column. This provides the basis for improved correlations between the results of the measurements performed in the solvent extraction columns 
and the uranium inventory in the pulser lines. These new correlations are discussed and evaluated in Sec. VI.A.l.

\section{B. Concentration Assays}

The asterisks plotted in Figs. 16-18 are the concentrations of uranium samples withdrawn from the static columns at the indicated height and assayed externally. In all cases, these samples were withdrawn from the columns before the static measurements but after the pulsing of the isolated columns for phase separation. The asterisks fall below the solid smooth curve that connects the static assay data in Fig. 16. The discrepancy between the smooth surve and the sample results is $10 \% \pm 4 \%(1 \sigma)$.

Several possible causes for these discrepancies have been investigated. Among these is the calibration of the concentration assay for each detector. The standard cell was moved out to the operations area in order to perform the direct calibration of each detector at its nomal location in the solvent extraction system. The calibration results (open circles in Fig. 13) show that the foil assays track the detector efficiencies to within $0.5 \%$, dismissing calibration of the concentration assay as the cause of the discrepancies.

Contamination on the outer surfaces of the columns was also dismissed as a possible cause by performing the EUREKA assays (on the static columns), decontaminating the outer surfaces of the columns, and running the assays again. Contamination of the inner surfaces of the columns with insoluble HEU was another possibility, but this was dismissed when the inventory results following April operations (Figs, 16-18) showed agreement between the smooth curve drawn between the static EUREKA assay results and the results of the aqueous sample assays (asterisks).

To date, the cause of the discrepancies has not been identified. The average magnitude of the difference is substantially larger than the average bias $(0.6 \%)$ in the EUREKA column inventory results. The smoothness in the EUREKA concentration vs column height reduces the possibility that a bias in the calibration of a single detector (detector 4, for example, which is adjacent to the aqueous sample spigot on all three columns) could be responsible for discrelancies of this magnitude. The origin of the observed discrepancies is probably in the sampling of the columns or in false assumptions about the sampling (for example, plotting the sample assay results at the height of the sample spigot). 
Because uranium is partitioned between two immiscible phases in the solvent extraction columns, a gradient in concentration vs column height will always exist within a phase, even after long periods of pulsing following shutdown and isolation of the columns. This gradient, which can cause a continuous change in the uranium concentration profile in the column, contributes to the difficulty of using sampling methods to evaluate the EUREKA concentration assay. For the same reason, determination of HEU inventory in the solvent extraction columns by sampling is subject to large uncertainties. The choice of the on-line NDA methods applied in the EUREKA instrument was made, in part, to overcome the uncertainties in the inventory results introduced by sampling. The concentration monitor on the $\mathrm{Y}-12$ intermediate evaporator return loop offers a more reasonable situation for evaluation of the concentration assay by sanspling because only a single-phase solution is present in the evaporator.

\section{Inventories Obtained During Pulsed Operation}

The EUREKA instrument routinely assays uranium concentration during pulsed operation, primarily to deduce the uranium concentrations (and hence the HEU inventory) in the plumbing external to the secondary solvent extraction system. Because the uranium concentration profile is measured during pulsed operation by the EUREKA detectors, the results of these assays can al so be used to deduce the HEU inventory in the operating columns. Furthermore, if steady-state operation of the columns is achieved at the time of the EUREKA assays during pulsed operation and if steady state persists until the time of shutdown, then the EUREKA pulsed and static inventories should be the same.

The use of the assay data obtained during pulsed operation for determination of the HEU inventory in the solvent extraction columns is appealing because it opens the possibility of using a computerized chemical mode ${ }^{8}$ to give an accurate concentration profile that requires fewer than six concentration measurements (that is, six detectors) per column. The success of such a model, however, requires steady-state operation of the solvent extraction system.

Table XVI is a tabulation of the HEU inventories in the three columns determined from the pulsed and static EUREKA assays for the four inventory periods. The quantity $\Delta T$ is the time elapsed between the pulsed assays and the actual column shutdown. The largest differences between static and pulsed inventories appear in the February results, which correspond to the longest time 
TABLE XVI

EUREKA PULSED AND STATIC COLUMN INVENTORIES (GRAMS U)

\begin{tabular}{|c|c|c|c|c|c|}
\hline DAIE & $\begin{array}{c}\text { COLUHA } \\
\text { ID }\end{array}$ & PULSED & SIAUIC & $\frac{\text { SIAIIC }}{\text { PIIISED }}$ & SI(HOURS) \\
\hline \multirow[t]{4}{*}{$2 / 4 / 83$} & EXTR. & 7026 & 9017 & 1.28 & 20 \\
\hline & STRIP & 7916 & 9156 & 1.16 & 20 \\
\hline & BACKW. & 2148 & 4339 & 2.02 & 20 \\
\hline & TOTAL & 17090 & 22512 & 1.32 & \\
\hline \multirow[t]{4}{*}{$3 / 4 / 83$} & EXIR. & 6573 & 6497 & 0.99 & 1.5 \\
\hline & STRIP & 8666 & 7955 & 0.92 & 0.5 \\
\hline & BACKW. & -3372 & 3148 & 0.93 & 0.25 \\
\hline & TOTAL & 18611 & 17600 & 0.95 & \\
\hline \multirow{4}{*}{$4 / 8 / 83$} & EXTR. & 8780 & 9103 & 1.04 & 5 \\
\hline & SIRIP & 8061 & 8658 & 1.07 & 5 \\
\hline & BACKW. & 3516 & 3650 & 1.04 & 5 \\
\hline & TOTAL & 20357 & 21411 & 1.05 & \\
\hline \multirow[t]{4}{*}{$5 / 5 / 83$} & EXTR. & 5345 & 3847 & 0.72 & 3 \\
\hline & STRIP & 9093 & 9357 & 1.03 & 3 \\
\hline & BACKW. & 3152. & 4039 & 1.28 & 3 \\
\hline & TOTAL & 17590 & 17243 & 0.98 & \\
\hline \multirow[t]{4}{*}{$8 / 4 / 83$} & EXTR. & 6896 & 4634 & 0.67 & 18 \\
\hline & STRIP & 8582 & 9442 & 1.10 & 18 \\
\hline & BACKW. & 5143 & 3760 & 2.73 & 18 \\
\hline & TOTAL & 20621 & 17836 & 0.86 & \\
\hline \multirow[t]{4}{*}{$2 / 5 / 83$} & EXTR. & 9437 & 8920 & 0.95 & 60 \\
\hline & STRIP & 8718 & 8659 & 0.99 & 60 \\
\hline & BACKW. & -3420 & 3295 & 0.96 & 60 \\
\hline & TOTAL & 21575 & 20874 & 0.97 & \\
\hline
\end{tabular}

delay between pulsed assays and shutdown; but substantial differences al so exist in the May results for which the delay was only $3 \mathrm{~h}$.

Production routines are such that there can be no guarantee of steadystate operation of the solvent extraction systems. Furthermore, the routine procedure of adding acid (or deionized water) to the columns after shutdown and phase separation in order to raise the interface to a height above the glass/ stainless steel flange alters the column HEU inventory from that at shutdown. Therefore, the ability to use the concentration assays in the pulsed mode (and hence the usefulness of a chemical model in simplifying the EUREKA inventory determination) cannot be evaluated using the results obtained in these exercises. The use of empirical models that correlate inventory with the concentration assay at one or two critical locations on the column may be a more 
reasonable approach under the present operating conditions. Additional pulsed EUREKA assay data are required for an evaluation of empirical correlations.

\section{System Inventory Resul ts}

The HEU inventories deduced by EUREKA for the plumbing external to the solvent extraction columns show a deviation from the dump results of $11 \%$ for the average plumbing totals [Table XV(A)]. Because the plumbing inventory is approximately $17 \%$ of the total inventory, the additional bias introduced into the total EUREKA inventory is $1.7 \%$. Thus, the average deviation of the EUREKA system inventory totals from the direct results is $2.9 \%$ (compared with $1.2 \%$ for the column totals). The standard deviation in the relative results for the EUREKA plumbing inventory totals is $14.6 \%$. This increases the standard deviation in the relative results for the system inventory totals from $2.3 \%$ (for the column totals) to $4.2 \%$.

Some of the $11 \%$ deviation and much of the $14.6 \%$ scatter in the plumbing results for EUREKA relative to the dumps must be attributed to changes in concentrations in feed and product streams between the time of the pulsed EUREKA assays and the shutdown. These are due to lack of steady-state operating conditions. Furthermore, $4.2 \%$ of the average plumbing deviation is the result of an overestimate by EUREKA of the solution volumes in the external plumbing [Table XIII(A)]. The volumes assumed by EUREKA are obtained by calculations using the known diameters and lengths of pipe in the external plumbing. It is assumed that these plumbing volumes are full of solution at inventory, but for some of the external components, this appears to be a false assumption.

For purposes of the operational evaluation, the average deviation in the relative totals of the EUREKA solvent extraction system inventory is $2.9 \%$ and the standard deviation in the relative results is $4.3 \%$. Reductions in the average deviation can be achieved by using more realistic volumes for the external plumbing components. However, the largest reductions in the average deviation and substantial improvements in the standard deviation are achieved with the use of new plumbing correlations that do not rely on the EUREKA results during pulsed operation, but rather on the HEU inventories and concentrations in the static columns. These are better indicators of the operation parameters of the solvent extraction system at the time of shutdown (and hence correlate better with the plumbing inventories) than the concentrations 
measured during pulsed operation. Section VI.A.1 gives details on the choice of the new correlations and on the improvements achieved and provides recommerdations for future operations of EUREKA.

\section{DISCUSSION}

\section{A. EUREKA}

1. Improvements in System Inventory. The nonsteady-state operating conditions of the secondary solvent extraction system and the partial draining of the external plumbing at the time of shutdown are the major causes of large errors in the EUREKA inventory results for the plumbing external to the solvent extraction columns. These errors propagate to considerably smaller but nonetheless significant relative errors in the total system inventory.

The adjustment of the external plumbing volumes to match the empirical results obtained from the dumps is a straightforward approach that addresses one of these sources of error. However, the approach used by the original EUREKA instrument for determination of the HEU inventory in the external plumbing requires both the plumbing solution volumes and the uranium concentrations. It is unlikely that steady-state operating conditions will be achieved under the present conditions of solvent extraction system operation. Because of the difficulties of scheduling production routines, it is unlikely that the EUREKA assays can be performed during pulsed operation within minutes of the actual time of shutdown. (This occurred only once in the six inventory periods documented in Table XVI.) Under these circumstances, concentrations measured by EUREKA during pulsed operation will not be representative of concentrations in the plumbing lines at the time of shutdown. Therefore, it is unlikely that the EUREKA plumbing inventories can be improved significantly using the original EUREKA approach.

Another approach to obtaining the external plumbing inventories is to assume that the HEU concentrations and inventories measured by EUREKA for the static columns are determined by the operation parameters of the solvent extraction system at the time of shutdown. Therefore, there should be a correlation between these concentrations and inventories and the HEU inventories in the external plumbing. The simplest correlation assumes that the relevant operation parameters (for a particular plumbing line) and, therefore, the HEU inventory (for the particular plumbing line) are constant. 
Six correlations were evaluated for determinatior of the contribution of each column to the aqueous plumbing invenicory. These are

i. $k$,

ii. $K \cdot I_{A Q}$,

iii. $K \cdot \bar{\rho}_{A Q}$,

iv. $K \cdot I_{\text {TOT }}$,

v. $K \cdot \rho_{4}$, and

vi. $K \cdot \rho_{1}$,

where $K$ is a constant; $I_{A Q}$ and I $I_{T O T}$ are the EUREKA inventories of the aqueous phase and of both aqueous and organic phases, respectively, of the static column; $\bar{\rho}_{A Q}$ is the average concentration of uranium in the aqueous phase of the static column (that is, I $A Q$ divided by the volume of the aqueous phase); and $\rho_{1}$ and $\rho_{4}$ are the uranium concentrations measured by detectors 1 and 4 , respectively, during the EUREKA assay of the static column. Correlations i-iv were also evaluated for determination of the contribution of each column to the organic plumbing inventory. For determination of the HEU inventory in the pulser line of each column, correlations $i, i i, v i$, and

$$
\text { vii. } K \cdot \rho_{i} \cdot I_{\text {TOT }}
$$

were evaluated. Table XVII gives the correlations that achieved the best overall agreement with the dump results for each plumbing component.

TABLE XVII

NEW PLUMBING CORRELATIONS (GRAMS U)

\begin{tabular}{|c|c|c|c|}
\hline \multirow{2}{*}{$\begin{array}{l}\text { PLUMBING } \\
\text { COMPONENI }\end{array}$} & \multicolumn{3}{|c|}{ COLUMN } \\
\hline & EXIRACIION & SIRIP & BACKWASH \\
\hline ORGANIC & 1584 & 1044 & 118 \\
\hline AQUEOUS & $0.0506 \cdot I_{A 0}$ & 335 & $0.1653 \cdot I_{A}$ \\
\hline PULSER & $0.2204 \cdot p_{1} \cdot I_{\text {TOT }}$ & $2441 \cdot A_{1}$ & 2602 \\
\hline
\end{tabular}

$I_{A O}=$ STATIC COLUMN INVENTORY OF AQUEOUS PHASE (GRAMS)

$I_{\text {TOT }}=$ TOTAL STATIC COLUMN INVENTORY (GRAMS)

$p_{I}=$ DETECTOR 1 SIATIC CONCENTRATION ASSAY (GRAMS PER MILLILITER) 
The organic plumbing inventories were reproduced best for all three columns by a constant (correlation $i$ ). The reason is that, despite variations in the column operation parameters, the organic phase HEU concentration is close to saturation in the extraction and strip columns and is very low in the backwash column during operation. A constant also gave the best result for the HEU inventory in the aqueous plumbing of the strip column. This is probably the result of the constant concentration of the organic feed (that is, the organic product of the extraction column) and of the aqueous feed (that is, the refluxed portion of the aqueous product solution) to the strip column.

Because of large variations in the aqueous feed concentration and in some operation parameters (for exaniple, flow rates) of the extraction column, the aqueous plumbing inventory from the extraction column varies by an order of magnitude. The quantities $I_{A \cap}, I_{T O T}, \rho_{A}, \rho_{1}$, and $\rho_{4}$ are all sensitive to these variations. However, $\rho_{1}$ and $\rho_{4}$ are also sensitive to the duration of pulsing for phase separation after shutdown. Therefore, the best correlations for the aqueous plumbing inventory for the extraction column were $i i, i i i$, and $i v$. Of these, the $I_{A \cap}$ dependence (correlation $i i$ ) gave the best results for the extraction column and also for the backwash column aqueous plumbing components.

For all three columns, the HEU inventory in the pulser line is correlated most strongly with $\rho_{1}$. Therefore, correlation vi gave the best results for the pulser line inventories of the strip and backwash columns. However, because of large variations in the HEU inventory in the extraction column (the inventories in the strip and backwash columns are relatively sonstant) and because of larger gradients in concentration in the extraction column, the optimal correlation for the extraction column pulser ine inventory includes a dependence on ITOT' as well as on $\rho_{1}$ (correlation vii), for reasons discussed in Sec. V.A.1.

The new plumbing correlations (NPCS) given in Table XVII were used to redetermine the EUREKA inventory results previously given in Table XI. The NPC inventory results are given in Tabie XVIII. Tables $X I X(A)$ and $X I X(B)$ give the ratios of the EUREKA HEU inventory results obtained with the NPCs to the reference inventory values for the directly measured dump components. (These are the ratios of the quantities in Table XVIII to those in Table IX.) The means and standard deviations in these ratios are given in Tables $X X(A)$ and $X X(B)$. 
TABLE XVIII

EUREKA NPC INVENTORY RESULTS (GRAMS U)

\begin{tabular}{|c|c|c|c|c|c|}
\hline \multirow[b]{2}{*}{ DAIE } & \multirow{2}{*}{$\begin{array}{c}\text { COLUMA } \\
\text { ID }\end{array}$} & \multicolumn{4}{|c|}{ COMPONENT } \\
\hline & & Columa & PULLSER & PLumbing & IOIAL \\
\hline \multirow[t]{4}{*}{$2 / 4 / 83$} & EXTR. & 9017 & 255 & 1971 & 11243 \\
\hline & STRIP & 9156 & 539 & 1379 & 11074 \\
\hline & BACKW. & 4339 & 335 & 780 & 5454 \\
\hline & TOTAL & 22512 & 1129 & 4130 & 27771 \\
\hline \multirow[t]{4}{*}{$3 / 4 / 83$} & EXTR. & 6497 & 146 & 1900 & 8543 \\
\hline & SIRIP & 7955 & 433 & 1379 & 9767 \\
\hline & BACKW. & -3148 & 255 & 621 & 4024 \\
\hline & TOTAL & 17600 & 834 & 3900 & 22334 \\
\hline \multirow[t]{4}{*}{$5 / 5 / 83$} & EXTR. & 3847 & 13 & 1742 & 5602 \\
\hline & SIRIP & 9357 & 446 & 1379 & 11182 \\
\hline & BACKW. & 4039 & 292 & 749 & 5080 \\
\hline & TOTAL & 17243 & 751 & 3870 & 21864 \\
\hline \multirow[t]{4}{*}{ 9/7/83 } & EXTR. & 10074 & 355 & 2045 & 12474 \\
\hline & STRIP & 9756 & 534 & 1379 & 11669 \\
\hline & BACKW. & 3803 & 303 & 657 & -4763 \\
\hline & TOTAL & 23633 & 1192 & 4081 & 28906 \\
\hline \multirow[t]{4}{*}{$12 / 5 / 83$} & EXIR. & 8920 & 252 & 1986 & 11158 \\
\hline & STRIP & 8659 & 505 & 1379 & 10543 \\
\hline & BACKW. & 3295 & 286 & 638 & 4219 \\
\hline & TOTAL & 20874 & 1043 & 4003 & 25920 \\
\hline
\end{tabular}

The average deviation in the EUREKA external plumbing HEU inventory totals relative to the direct results is reduced from $11 \%$ [Table XV(A)] to $-2.9 \%$ [Table $X X(A)]$, and the standard deviation drops from $14.6 \%$ [Table XV(A)] to $7.9 \%$ [Table $X X(A)]$ with the NPCs. The average deviation in the relative totals of the EUREKA system inventory is $1.2 \%$, and the standard deviation is $2.4 \%$ with the NPCs [Table XX(A)]. Improvements in the EUREKA inventory relative totals for the pulser lines include large reductions in the average deviation (from $-16 \%$ to $-0.7 \%$ with the NPCs) and in the standard deviation (from $22.1 \%$ to $0.4 \%$ with the NPCS), as indicated by the totals in Tables $X V(B)$ and $X X(B)$.

Operational procedures for evaluation of the EUREKA inventory measurements evolved during the evaluation. Therefore, toward the latter part of the evaluation, the procedures for obtaining the reference values (by the dumps) were more controlled and also better designed to give valid reference values compared with practices applied earlier in the evaluation. This must account for some of the reductions in the average deviations and standard deviations in 
TABLE $X I X(A)$

(EUREKA NPC INVENTORY)/(DUHP INVENTORY)

\begin{tabular}{|c|c|c|c|c|}
\hline \multirow[b]{2}{*}{ DAIE } & \multirow[b]{2}{*}{ COLUAN } & \multicolumn{3}{|c|}{ COMPONENI } \\
\hline & & COLUHA \pm PULSER & PLUEBING & IOTAL \\
\hline \multirow[t]{4}{*}{$2 / 4 / 83^{*}$} & EXTR. & 0.982 & 0.895 & 0.966 \\
\hline & STRIP & 1.052 & 0.904 & 1.031 \\
\hline & BACKW. & 1.070 & 1.024 & 1.063 \\
\hline & TOTAL & 1.027 & 0.920 & 1.009 \\
\hline \multirow[t]{4}{*}{$3 / 4 / 83=$} & EXTR. & 1.119 & 1.216 & 1.139 \\
\hline & STRIP & 0.993 & 1.084 & 1.005 \\
\hline & BACKW. & 0.967 & 0.878 & 0.952 \\
\hline & TOTAL & 1.030 & 1.101 & 1.041 \\
\hline \multirow[t]{4}{*}{$5 / 5 / 83 * *$} & EXTR. & 1.154 & 1.071 & 1.127 \\
\hline & SIRIP. & 1.019 & 0.927 & 1.007 \\
\hline & BACKW. & 1.013 & 0.894 & 0.994 \\
\hline & TOTAL & 1.044 & 0.979 & 1.032 \\
\hline \multirow[t]{4}{*}{$9 / 7 / 83^{* n}$} & EXTR. & 1.016 & 0.825 & 0.978 \\
\hline & SIRIP & 0.995 & 1.045 & 1.001 \\
\hline & BACKW. & 0.999 & 1.254 & 1.028 \\
\hline & TOTAL & 1.004 & 0.944 & 0.995 \\
\hline \multirow[t]{4}{*}{$12 / 5 / 83^{*}$} & EXTR. & 1.015 & 0.859 & 0.984 \\
\hline & STRIP & 0.986 & 0.959 & 0.982 \\
\hline & BACKW. & 0.986 & 0.991 & 0.986 \\
\hline & TOTAL & 0.998 & 0.911 & 0.983 \\
\hline
\end{tabular}

TABLE XIX(B)

(EUREKA NPC INVENTORY)/(DUMP INVENTORY)*

\begin{tabular}{llll}
\multirow{2}{*}{ DAIE } & COLUHN & \multicolumn{2}{c}{ COMPONENT } \\
\hline \multirow{2}{*}{$5 / 5 / 83$} & COLUMH & PULSER \\
& EXTR. & 1.155 & 1.000 \\
& STRIP & 1.022 & 0.967 \\
& BACKW. & 1.013 & 1.025 \\
& TOTAL & 1.046 & 0.989 \\
$9 / 7 / 83$ & & & \\
& EXTR. & 1.016 & 1.000 \\
& STRIP & 0.992 & 1.043 \\
& BACKW. & 1.006 & 0.921 \\
& TOTAL & 1.005 & 0.997 \\
& & & \\
$12 / 5 / 83$ & EXTR. & 1.014 & 1.054 \\
& STRIP & 0.988 & 0.953 \\
& BACKW. & 0.983 & 1.018 \\
& TOTAL. & 0.997 & 0.993
\end{tabular}

"ANALYSIS OF RESULTS FROM LAST THREE INVENTORY PERIODS WHEN PULSER COMPONENIS WERE
DUMPED SEPARATEI.Y FROM COLUHN COMPONENTS.

* COLUMA AND PULSER CONTENTS DUMPED SIMUL TANEOUSLY

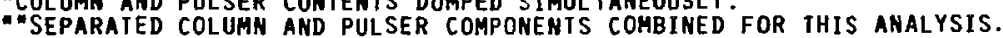


TABLE $X X(A)$

AVERAGE VALUES (\$10)* OF (EUREKA NPC INUENTORY)/(DUMP INVENTORY)

\begin{tabular}{|c|c|c|c|}
\hline $\begin{array}{l}\text { COLUMN ID } \\
\text { (NO.AVERAGED) }\end{array}$ & COLUHK + PULSER" & PLUMBUNG & TOTAL \\
\hline EXTR. (5) & $1.057(7.0 x)$ & $0.973(17.18)$ & $1.039(8.3 \%)$ \\
\hline STRIP (5) & $1.009(2.7 x)$ & $0.984(7.8 x)$ & $1.005(1.7 \%)$ \\
\hline BACKH. (5) & 1.007 (3.9\%) & $1.008(15.0 \%)$ & $1.005(4.2 \%)$ \\
\hline (15) & $1.024(5.2 x)$ & $0.988(12.9 \times)$ & $1.016(5.4 \%)$ \\
\hline$(14)^{n * n}$ & $1.014(3.9 x)$ & $0.983(13.2 x)$ & $1.009(4.7 x)$ \\
\hline TOTALS (5) & $1.020(1.9 x)$ & $0.971(7.9 x)$ & $1.012(2.4 x)$ \\
\hline
\end{tabular}

- SAMPLE RELATIVE STANDARD DEVIATION.

* SEPARATEd Column and PULSER components combined for this analysis.

" "OMIT 5/5/83 EXTRACTION COLUMN RESULT ON STATISTICAL GROUNDS.

TABLE $\times X(B)$

AVERAGE VALUES (\$10)* OF (EUREKA NPC INVENTORY)/(DUMP INVENTORY)**

\begin{tabular}{|c|c|c|}
\hline $\begin{array}{l}\text { COLUMN ID } \\
\text { SWO AVERAGED) }\end{array}$ & COLUMN. & PULSER \\
\hline EXTR. (3) & $1.061(7.6 x)$ & $1.018(3.0 x)$ \\
\hline STRIP (3) & $1.001(1.97)$ & $0.988(4.9 x)$ \\
\hline BACKW. (3) & $1.001(1.6 x)$ & $0.988(5.94)$ \\
\hline ALL $\quad$ (9) & $1.021(5.1 x)$ & $0.998(4.4 x)$ \\
\hline$(8) \cdots *$ & $1.004(1.5 x)$ & $0.998(4.7 x)$ \\
\hline TOTALS(3) & $1.013(2.3 x)$ & $0.993(0.4 x)$ \\
\hline
\end{tabular}

" Sample relative standard deViation.

- Last thREE INVENTORY PERIODS WHEN PULSER COMPONENTS WERE DUMPED SEPARATELY FROM COLUMN COMPONENTS

" "OMIT $5 / 5 / 83$ EXTRACTION COLUMN RESULT ON STATISTICAL GROUNDS. 
Tables $X X(B)$ or $X V(B)$ compared with Tables $X X(A)$ or $X V(A)$. The most outstanding of these is the reduction in the standard deviation in the column plus pulser column-by-column inventory ratios [ $24 \%$ for the 14 measurements as given in Tables $X X(A)$ and $X V(A)]$ compared with the standard deviation in the three most recent column inventory comparisons, column by column $[1.5 \%$ for the 8 measurements in Tables $X X(B)$ and $X V(B)]$. The more recent results of $1.5 \%$ indicate that the actual random uncertainty in the EUREKA column inventory could be approaching the predicted minimum of $1 \%$ (Sec. II.C) because the observed $1.5 \%$ also includes the known $0.7 \%$ minimum contribution of the reference values for column inventory to the random uncertainty (Sec. III.B).

2. Evaluation of the Uncertainty Introduced by the Histogram Method. The construction of a histogram from the EUREKA static concentration profile data (concentration vs column height) is the most simple, systematic approach to the determination of the column HEU inventories. The inventory is the area under the curve that plots concentration vs volume where volume is a function of vertical height along the column. Because the static inventories are obtained from measurements of nonequilibrated two-phase systems, the shapes of the profiles (that is, uranium concentration vs height) vary tremendously depending on the degree of equilibration of concentration, on the HEU loading, and on relative phase volumes. This is most apparent in the extraction column in which the loading varies from 3 to $10 \mathrm{~kg}$. Therefore, it is uifficult to select a function to which the profile data can be fitted for purposes of integration; consequently, the histogram method was used.

An alternate approach to integration of the profile information is the use of spline interpolation procedures to systematically construct smooth curves between the data points. In a strict sense, the curves resulting from spline interpolations of six points are not physically realistic. In the same sense, the histograms do not represent the actual profile. However, because these are independent, systematic approaches to obtaining the column inventories, the variation in the ratio of the inventories obtained by the two methods and applied to the same data can be used as an upper limit for estimating the random uncertainties in inventories deduced by integration of the histograms.

Cubic spline interpolations were applied to the static EUREKA data obtained during the 1983 evaluation. The resulting inventories were computed, and these were compared, column by column, with the analogous results of the 
histogram method. The ratios of inventories obtained by the two methods are given in Table XXI.

On the average, the spline results show the largest deviation (positive) from the histogram results, as well as the widest variation, $1 \sigma$, relative to the histogram results $(1.4$ and $1.7 \%$, respectively) for the extraction column. This is due, in part, to overestimation by the spline interpolation of the curvature where the slope (of concentration vs volume) changes sign. However, even in this worst case, the $1.7 \%$ standard deviation is small relative to the scatter observed in the EUREKA-to-dump ratios. For both the strip and backwash columns, the agreement between the histogram and spline results, as well as the scatter in these ratios, is better than $1 \%$. Therefore, the histogram method contributes up to $1 \%$ to the actual random uncertainty in the EUREKA column inventories.

3. Summary and Recommendations. The EUREKA evaluation results have shown that the average deviation of the EUREKA inventory results from the direct measurements of dumped components is $1.2 \%$ [Table $X X(A)]$ when optimal correlations for determination of the plumbing inventories are applied. The evaluation al so shows that the random uncertainty in the EUREKA system inventory is $<2.4 \%$ because the measured standard deviation of $2.4 \%$ in the relative system

TABLE XXI

INVENTORY (HISTOGRAM)/INVENTORY (SPLINE)*

\begin{tabular}{|c|c|c|c|c|}
\hline $\begin{array}{l}1983 \\
\text { INVENTORY }\end{array}$ & & & & \\
\hline PERION & EXIRACIION & STRIP & BACKWASH & TOIAL \\
\hline JANUARY & 0.983 & 0.988 & 0.993 & 0.987 \\
\hline FEBRUARY & 0.994 & 0.998 & 1.001 & 0.997 \\
\hline MARCH & 0.983 & 0.989 & 1.011 & 0.990 \\
\hline APRIL & 1.004 & 0.994 & 1.002 & 0.998 \\
\hline JULY & 1.020 & 0.994 & 0.996 & 1.001 \\
\hline AUGL'ST & 0.968 & 0.985 & 0.987 & 0.978 \\
\hline SEP TEMBER & 0.974 & 0.992 & 0.986 & 0.982 \\
\hline OC TOBER & 0.971 & 0.986 & 1.000 & 0.982 \\
\hline NOVEMBER & 0.977 & 0.991 & 1.011 & 0.988 \\
\hline MEAN & 0.986 & 0.991 & 0.999 & 0.989 \\
\hline 10. DATA & 0.017 & 0.004 & 0.009 & 0.008 \\
\hline
\end{tabular}

"AVERAGE OF 27 ENTRIES $=0.992$

(10, DATA $=0.012)$ 
inventory totals [Table $X X(A)$ ] inciudes the random uncertainty in the reference values for the dumped components. The column-by-column comparisons of column inventory results for EUREKA and the dumps [Table $X V(B)$ or $X X(B)]$ show that the random uncertainty in the EUREKA result based on the most recent measurements is $1.5 \%$ (because of a $0.7 \%$ minimum contribution by the reference values for column inventory) and therefore approaches the predicted minimum random uncertainty of $1 \%$ for the individual EUREKA column inventories.

Because the measurement control program has revealed that the detector efficiency factors vary with time, a routine measurement control program must be exercised to ensure that accuracy is not sacrificed. However, because the variations are monotonic and gradual, a substantial reduction is justified in the measurement control effort (from that exercised during operational evaluation) and therefore in the monthly inventory effort.

Use of EUREKA measurements obtained during pulsed operation to deduce the piumbing inventories should be abandoned and replaced by the NPCs that rely only on the static EUREKA results. This further reduces (to one-half) the required monthly inventory effort.

The withdrawal from the columns and external assay of aqueous and organic solution samples before performing the EUREKA measurements can be discontinued. The correction factors to the $188-\mathrm{keV}$ count rates because of plumbing internal to the glass sections have been determined using the organic sample assay results throughout the evaluation period. Furichermore, the default valuss used by the EUREKA code for acid molarity of the aqueous solutions are in sufficient agreement with those determined from the assay of the aqueous solution samples to eliminate this as a reason for continued sample withdrawal. Elimination of the need for external assay of solution samples further simplifies the monthly inventory effort.

Extension of the EUREKA measurements to the second (parallel) solvent extraction system is recommended as a final step in implementing the routine use of this instrument for HEU inventory of the static system.

\section{B. Evaporator Monitor}

The uranium concentration monitor for the $\gamma-12$ secondary intermediate evaporator has performed accurately and reliably since its installation in December 1982. Because these performance criteria have been satisfied, the possibility of automatic control of the evaporator operation can now be 
considered. The desired product solution for feed into the secondary solvent extraction system is well defined in uranium concentration, as well as in acid molarity. For this reason, combined on-line measurements of uranium concentration and specific gravity might be used as a basis for automating control of the evaporator to achieve the optimal product.

Improvements in the precision of a single monitor assay have been achieved by reducing the monitor deadtime by performing data acquisition and conputation functions simultaneously. The predicted random uncertainty in the 1-min assay is now $3 \%$ (compared with $5 \%$ ).

The routine measurement control procedures exercised during the operational evaluation of the evaporator monitor provide the only mechanism for as suring the continued reliability of the results provided by this on-line monitor. Continuation of these procedures is essential.

\section{ACKNOWLEDGMENTS}

Controlled evaluation of in-line, in-plant instrumentation requires substantial effort by the facility operator. These in-1ine instruments at $\mathrm{Y}-12$ have been designed so that routine use does not impact the process operation routines and schedules. However, the success of the extensive characterization of EUREKA and the evaporator monitor performance throughout calendar year 1983 is a direct result of the cooperation of personnel from Chemical Processing, namely, Herman Butler and his staff at the Y-12 scrap recovery facility.

The development of prototype instrumentation requires dedication to design beyond fabrication of the original (hardware, software, and electronics) product. The continued design support of Ted Dye, David L. Garcia, Susan Johnson, and Roy Slice of Los Alamos have contributed to the success of this evaluation.

The resources and support of the Nuclear Materials Control and Accounting Department at Y-12 and the Safeguards Assay Group at LoS Alamos were made available for the duration of this project by Bill Mee and John Foley. Momentum for the joint undertaking of this project by $Y-1$ ? and Los Alamos was provided by Roddy B. Walton. 


\section{REFERENCES}

1. P. A. Russo and R. B. Strittmatter, "On-Line Prototype NDA Instruments for Material Accounting and Monitoring of HEU Recovery Processes," Trans. Am. Nuc1. Soc. 43, 274 (1982).

2. I. W. Jeter, S. E. Smith, H. H. Hogue, G. L. Bowers, P. A. Russo, and R. B. Strittmatter, "Applications of NDA Instrumentation in the $\mathrm{Y}-12$ Highly Enriched Uranium Recovery Facility," Proceedings of Orlando International AICHE Meeting, Orlando, Florida (1982), available in microfiche from AICHE.

3. P. A. Russo, R. B. Strittmatter, E. L. Sandford, I. W. Jeter, E. McCu1lough, and G. L. Bowers, "Operation of Automated NDA Instruments for InLine HEU Accounting at $\gamma-12$," Proceedings of American Nuclear Society Conference on Safeguards Technology: The Process-Safeguards Interface, Hilton Head Island, South Carolina, November 28-December 2, 1983, USDOE New Brunswick Laboratory, Conf. No. 831106 (November 1984), pp. 112-134.

4. L. Brumfield, S. E. Smith, E. E. Mccullough, and P. Russo, "On Line Operation of NDA Instrumentation on a Solvent Extraction System," Martin Marietta Energy System, Inc., Oak Ridge Y-12 Plant report Y/DG-16602 (1984).

5. J. R. Barkman, "A Chemical Recovery System for Safeguarding Nonirradiated Uranium," Union Carbide Corporation, Oak Ridge Y-12 Plant report Y-MA-3582 (July 1970).

6. S. T. Hsue and T. Marks, "Measurement Control Program for NDA Instruments," Proceedings of American Nuclear Society Conference on Safeguards Technology: The Process-Safeguards Interface, Hilton Head Island, South Carolina, November 28-December 2, 1983, USDOE New Brunswick Laboratory, Conf. No. 831106 (November 1984), pp. 42.2-425.

7. H. H. Hogue and S. E. Smith, "Off-Line Nondestructive Assay at a Lranium Recovery Facility," Proceedings of American Nuclear Society Conference on Safeguards Technology: The Process-Safeguards Interface, Hilton Head Island, South Carolina, November 28-December 2, 1983, USDOE New Brunswick Laboratory, Conf. No. 831706 (November 1984), pp. 265-274.

8. C. A. Ostenak and A. F. Cermak, "Comparison of Predicted and Measured Pulsed-Column Profiles and Inventories," Proceedings of American Nuclear Society Conference on Safeguards Technology: The Process-Safeguards Interface, Hilton Head Island, South Carolina, November 28-December 2, 1983, USDOE New Brunswick Laboratory, Conf. No. 831106 (November 1984), pp. $236-247$. 